This is an electronic reprint of the original article. This reprint may differ from the original in pagination and typographic detail.

Author(s): Giesbertz, Klaas; Gritsenko, O. V.; Baerends, E. J.

Title: $\quad$ Response calculations based on an independent particle system with the exact oneparticle density matrix: Excitation energies

Year: $\quad 2012$

Version:

Please cite the original version:

Giesbertz, K., Gritsenko, O. V., \& Baerends, E. J. (2012). Response calculations based on an independent particle system with the exact one- particle density matrix:

Excitation energies. Journal of Chemical Physics, 136(9), Article 094104.

https://doi.org/10.1063/1.3687344

All material supplied via JYX is protected by copyright and other intellectual property rights, and duplication or sale of all or part of any of the repository collections is not permitted, except that material may be duplicated by you for your research use or educational purposes in electronic or print form. You must obtain permission for any other use. Electronic or print copies may not be offered, whether for sale or otherwise to anyone who is not an authorised user. 


\section{A The Journal of Chemical Physics}

\section{Response calculations based on an independent particle system with the exact one- particle density matrix: Excitation energies}

K. J. H. Giesbertz, O. V. Gritsenko, and E. J. Baerends

Citation: The Journal of Chemical Physics 136, 094104 (2012); doi: 10.1063/1.3687344

View online: http://dx.doi.org/10.1063/1.3687344

View Table of Contents: http://scitation.aip.org/content/aip/journal/jcp/136/9?ver=pdfcov

Published by the AIP Publishing

\section{Articles you may be interested in}

Response calculations based on an independent particle system with the exact one-particle density matrix:

Polarizabilities

J. Chem. Phys. 140, 18A517 (2014); 10.1063/1.4867000

Unrestricted density functional theory based on the fragment molecular orbital method for the ground and excited state calculations of large systems

J. Chem. Phys. 140, 144101 (2014); 10.1063/1.4870261

Excitation energies with linear response density matrix functional theory along the dissociation coordinate of an electron-pair bond in $\mathrm{N}$-electron systems

J. Chem. Phys. 140, 024101 (2014); 10.1063/1.4852195

Oscillator strengths of electronic excitations with response theory using phase including natural orbital functionals J. Chem. Phys. 138, 094114 (2013); 10.1063/1.4793740

Obtaining Hartree-Fock and density functional theory doubly excited states with Car-Parrinello density matrix search

J. Chem. Phys. 131, 204101 (2009); 10.1063/1.3266564

\section{AP APL Photonics \\ APL Photonics is pleased to announce Benjamin Eggleton as its Editor-in-Chief}

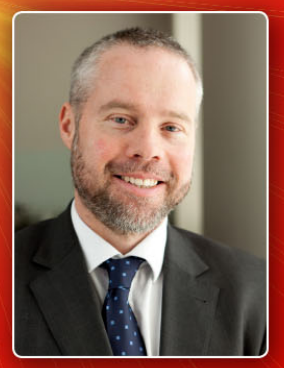




\title{
Response calculations based on an independent particle system with the exact one-particle density matrix: Excitation energies
}

\author{
K. J. H. Giesbertz, ${ }^{1,2,3}$ O. V. Gritsenko, ${ }^{2,3}$ and E. J. Baerends ${ }^{2,3,4, a)}$ \\ ${ }^{1}$ Nanoscience Center, P.O. Box 35, 40014 University of Jyväskylä, Finland \\ ${ }^{2}$ Theoretical Chemistry, VU University, De Boelelaan 1083, 1081 HV Amsterdam, The Netherlands \\ ${ }^{3}$ WCU Program, Department of Chemistry, Pohang University of Science and Technology, \\ Pohang, South Korea \\ ${ }^{4}$ Chemistry Department, Faculty of Science, King Abdulaziz University, Jeddah 21589, Saudi Arabia
}

(Received 21 November 2011; accepted 2 February 2012; published online 2 March 2012)

\begin{abstract}
Adiabatic response time-dependent density functional theory (TDDFT) suffers from the restriction to basically an occupied $\rightarrow$ virtual single excitation formulation. Adiabatic time-dependent density matrix functional theory allows to break away from this restriction. Problematic excitations for TDDFT, viz. bonding-antibonding, double, charge transfer, and higher excitations, are calculated along the bond-dissociation coordinate of the prototype molecules $\mathrm{H}_{2}$ and $\mathrm{HeH}^{+}$using the recently developed adiabatic linear response phase-including (PI) natural orbital theory (PINO). The possibility to systematically increase the scope of the calculation from excitations out of (strongly) occupied into weakly occupied ("virtual") natural orbitals to larger ranges of excitations is explored. The quality of the PINO response calculations is already much improved over TDDFT even when the severest restriction is made, to virtually the size of the TDDFT diagonalization problem (only single excitation out of occupied orbitals plus all diagonal doubles). Further marked improvement is obtained with moderate extension to allow for excitation out of the lumo and lumo +1 , which become fractionally occupied in particular at longer distances due to left-right correlation effects. In the second place the interpretation of density matrix response calculations is elucidated. The one-particle reduced density matrix response for an excitation is related to the transition density matrix to the corresponding excited state. The interpretation of the transition density matrix in terms of the familiar excitation character (single excitations, double excitations of various types, etc.) is detailed. The adiabatic PINO theory is shown to successfully resolve the problematic cases of adiabatic TDDFT when it uses a proper PI orbital functional such as the PILS functional. (C) 2012 American Institute of Physics. [http://dx.doi.org/10.1063/1.3687344]
\end{abstract}

\section{INTRODUCTION}

The time-development of a system subject to a timedependent perturbation results in time-dependence of properties such as the electron density and the full one-particle reduced density matrix (1RDM). These properties exhibit diverging responses at frequencies of the perturbation corresponding to excitation energies of the system. This effect is the basis of response theory based determination of the excitation energies. Time-dependent density functional (response) theory (TDDFT) uses the response of the electron density (diagonal of the 1RDM). Although typically quite successful for low-lying excitations at equilibrium geometry, it has in its customary adiabatic formulation severe shortcomings for many types of excitations: diagonal double excitations (typically (homo $)^{2} \rightarrow$ (lumo) $^{2}$, see Refs. 1-4), off-diagonal double excitations (excitation to two different virtuals) occurring in many excited states above the very lowest ones, ${ }^{3-5}$ bonding orbital to antibonding orbital (bond breaking) excitations, ${ }^{6,7}$ and charge transfer (CT) excitations. ${ }^{8}$ It is known that these deficiencies stem from the use of the adiabatic approximation, and could in principle be solved by the application of

\footnotetext{
a) Author to whom correspondence should be addressed. Electronic mail: e.j.baerends@vu.nl.
}

frequency dependent kernels. These, however, are not available, and would also make the calculations much more expensive. We will demonstrate in this paper that using timedependent one-body reduced density matrix functional theory (TD1MFT) (Refs. 3, 4, 9, and 10) in the adiabatic approximation offers a formulation of response theory that has important advantages. It can essentially solve all the problematic TDDFT cases mentioned above.

In TDDFT the density and its time-dependent response are described by the Kohn-Sham (KS) spinorbitals which have integer ( 1 or 0 ) occupations. Because of the constant integer occupations of the time-dependent KS orbitals, only $n_{\text {occ }} \times n_{\text {virt }}$ occupied-virtual density matrix elements $\delta \gamma_{i a}(\omega)$, $i \leq N, a>N$ contribute to the response of the density $\delta \rho(\omega)$ to the frequency-dependent change of the external potential $\delta v(\omega)$. These elements represent single excitations of the ground-state determinant of the KS system. One can say that the density response, the key quantity of the response TDDFT, has a "single-excitation" structure. Due to this structure, only $n_{\text {occ }} \times n_{\text {virt }}$ single-electron excitations can be determined from the response equations in adiabatic response TDDFT. ${ }^{11}$ Apparently, double excitations cannot be described in this approach, ${ }^{1,2}$ and a striking example of this failure is the lowest ${ }^{1} \Sigma_{g}^{+}$excitation of $\mathrm{H}_{2}$, which is absent in the 
adiabatic TDDFT calculations. ${ }^{3,4}$ Furthermore, it is a general trend that TDDFT produces reasonable excitation energies $\omega_{\alpha}$ when the Kohn-Sham orbital energy difference $\omega_{\alpha}^{s}=\epsilon_{a}-\epsilon_{i}$ is already a good first approximation, which is often the case for single excitations in compact molecules or in compact molecular fragments. ${ }^{12,13}$ The cases in which the $\omega_{\alpha}^{s}$ estimate fails are also, as a rule, the problematic TDDFT cases. Examples are the vanishing energy of a single excitation in a bonding-antibonding orbital pair, such as the $1 \sigma_{g} \rightarrow 1 \sigma_{u}$ excitation to the lowest ${ }^{1} \Sigma_{u}^{+}$state (the $B$ state) in the stretched $\mathrm{H}_{2}$ molecule, ${ }^{6,7}$ and the much too low long-range CT excitations ${ }^{8,14}$ obtained with the current adiabatic TDDFT. In both cases the divergence of the exchangecorrelation (xc) kernel $f_{\mathrm{xc}}$ of TDDFT is required in the longrange limit in order to obtain the correct $\omega_{\alpha},{ }^{6,12,15}$ while the functions $f_{\mathrm{xc}}$ of standard adiabatic local density (ALDA) and generalized gradient (AGGA) approximations remain finite.

The 1RDM can be written in its spectral representation as

$$
\begin{aligned}
\gamma\left(\boldsymbol{x}, \boldsymbol{x}^{\prime} ; t\right) & :=\left\langle\Psi\left|\hat{\psi}_{H}^{\dagger}\left(\boldsymbol{x}^{\prime} t\right) \hat{\psi}_{H}(\boldsymbol{x} t)\right| \Psi\right\rangle \\
& =\sum_{k} n_{k}(t) \phi_{k}(\boldsymbol{x} t) \phi_{k}^{*}\left(\boldsymbol{x}^{\prime} t\right),
\end{aligned}
$$

where the eigenfunctions, $\phi_{k}(\boldsymbol{x} t)$, are called the natural orbitals (NOs) and acquire fractional occupations $0 \leq n_{k}(t)$ $\leq 1$ due to the Coulomb electron correlation. Note that Eq. (1) does not define the phases of the NOs, since we can multiply the NOs with an arbitrary phase factor, $\mathrm{e}^{i \beta(t)}$ which cancels in the spectral expansion. The response $\delta \gamma(\omega)$, the key quantity of the response TD1MFT, has a richer structure than the density response. It involves in principle the matrix elements $\delta \gamma_{k l}(\omega)$ of all (fractionally occupied) NOs. We will see that the responses in these matrix elements faithfully represent single and double excitations. The theory does not rely on a splitting of the matrix to be diagonalized (representing essentially the inverse of the response function) into orbital energy differences and a coupling part, as is the case in TDDFT, and it does not require diverging constituents to describe excitations in stretched $\mathrm{H}_{2}$ and $\mathrm{CT}$ excitations. ${ }^{4}$

The latest generation of 1MFT functionals describe fairly accurately the molecular ground states. This holds for the equilibrium geometry and bond dissociation energy ${ }^{16-20}$ as well as for the energy curves along bond dissociation coordinates. $^{21,22}$ This success opens the road to the correct description with adiabatic TD1MFT of the excited state energy surfaces for the above mentioned TDDFT failure cases. However, a serious obstacle is the fact, that with approximate explicit functionals of $\gamma$ for the electron-electron interaction energy, $W[\gamma]$, as well as with implicit functionals of $\gamma$ and explicit functionals of the NOs and their occupations $W\left[\left\{\phi_{k}, n_{k}\right\}\right]$, which do not depend on possible NO phases, we inevitably obtain a zero response of the NO occupations $\delta n_{k}(\omega)=\delta \gamma_{k k}(\omega)=0$ in the standard adiabatic approximation. ${ }^{4,10,23,24}$ This artifact impairs calculated excitation energies, especially for double excitations. This problem has been solved recently by extending the TD1MFT with variables that describe some 2 -matrix information. These can be treated as the phases of the one-particle states of the the- ory, which are the NOs, hence the name phase-including NO (PINO) response theory. ${ }^{25-27}$

In the present paper the PINO response theory is applied to calculation of excitation energies along the bond dissociation coordinates of the prototype molecules $\mathrm{H}_{2}$ and $\mathrm{HeH}^{+}$. The calculated excitations include all the TDDFT failure cases. In Sec. II the PINO response theory is summarized and the matrix diagonalization is derived that in the adiabatic approximation of the PINO theory affords the excitation energies as eigenvalues and the corresponding vectors of responses of the density matrix elements as eigenvectors. In Sec. III the density matrix - density matrix response function is analyzed. It can be related, in the Lehmann representation, to the transition density matrices for the excited states of the system. This allows a relation to be established between the eigenvector of the diagonalization corresponding to an excitation and the transition density matrix of the corresponding excited state. The advantage over TDDFT is that the response vector is not limited to an occupied-virtual structure, but more general responses $\delta \gamma_{p q}$ appear, where $p$, $q$ can refer to both occupied and unoccupied NOs. [We call the first $N$ (usually strongly) occupied NOs the "occupied" NOs and the remaining (usually weakly occupied) ones the "virtual" NOs.] In Sec. IV the physical meaning of the 1RDM response corresponding to an excited state $\Psi_{\alpha}$ is analyzed from its relation to the transition density matrices $\Delta \gamma^{R}(\alpha)$ belonging to the state $\Psi_{\alpha}$ with excitation energy $\omega_{\alpha}$. The transition density matrix is defined as $\Delta \gamma^{R}\left(\alpha \mid 1,1^{\prime}\right)$ $:=N \int \Psi_{\alpha}(1,2, \ldots, N) \Psi_{0}^{*}\left(1^{\prime}, 2, \ldots, N\right) \mathrm{d} 2 \cdots \mathrm{d} N$. The ground state $\Psi_{0}$ and the excited states $\Psi_{\alpha}$ are supposed to be expanded in determinants in the NO basis, and the contributions to $\Delta \gamma^{R}(\alpha)$ from the matrix elements between basic determinants can be easily evaluated with the Slater-Condon rules for determinantal matrix elements. $\Psi_{0}$ can either be well approximated with the reference determinant $\Phi_{0}$ built from the first $N$ (strongly occupied) NOs, or it will have, in strong correlation cases, a significant admixture of a diagonal double excitation, usually of the type (homo) $)^{2} \rightarrow$ (lumo) $^{2}$. An examination of the commonly encountered cases for the ground state wavefunction (either single-reference type with one leading determinant or correlated with more determinants contributing) and of the excited state wavefunctions (single-excitation type or important contributions from diagonal double excitations or off-diagonal double excitations) affords a straightforward key to interpret the structures of the response vectors and transition density matrices in terms of single and double excitation character of the electronic transitions. In particular, nonzero $\delta \gamma_{i i}$ $=\delta n_{i}$ and $\delta \gamma_{a a}=\delta n_{a}$ contributions signify diagonal double excitation character, $(i i)^{2} \rightarrow(a a)^{2}$. Elements $\delta \gamma_{i a}$ represent single excitations (from occupied $i$ to unoccupied $a$ ) and $\delta \gamma_{a b}$ signifies off-diagonal double excitation (to the virtuals $a$ and $b$ ). The possibility of this identification is key to the improvement afforded by density matrix response theory compared to TDDFT: all these more general excitation types are covered.

Section $\mathrm{V}$ discusses the excitations to ${ }^{1} \Sigma_{u}^{+}$type along the bond dissociation coordinate of $\mathrm{H}_{2}$. They demonstrate that the TDDFT problem with bonding to antibonding 
excitation is solved with the PINO response theory. In Sec. VI the lowest ${ }^{1} \Sigma_{g}^{+}$excitations in $\mathrm{H}_{2}$ are presented. The lowest excited state of this symmetry, the $E F$ state $2^{1} \Sigma_{g}^{+}$, is for a large range of distances an essentially doubly excited state (to $\left(1 \sigma_{u}\right)^{2}$ configuration). The calculations demonstrate that such diagonal double excitations can be accurately calculated. The higher ${ }^{1} \Sigma_{u}^{+}$and ${ }^{1} \Sigma_{g}^{+}$states provide examples of important off-diagonal excitation character, which also are obtained accurately. In Sec. VII the lowest $\mathrm{CT}^{1} \Sigma^{+}$excitations in $\mathrm{HeH}^{+}$are presented. They demonstrate the feasibility of charge transfer transitions in the present scheme.

In principle the matrix diagonalization problem has much higher dimension than in the case of TDDFT. In the latter case it has dimension $n_{\text {occ }} n_{\text {virt }} \times n_{\text {occ }} n_{\text {virt }}$. In the TD1MFT (and PINO response theory as well) it has in principle roughly dimension $\frac{1}{2} n^{2} \times \frac{1}{2} n^{2}$, since all matrix elements $\delta \gamma_{p q}, p \geq q$ may appear in the response vector ( $n$ is the size of the basis set). However, we will see that the size of the problem can be reduced without significant loss of accuracy. The diagonal elements $\delta \gamma_{p p}=\delta n_{p}$ are always all included (although they can possibly be reduced), since this is only a set of order $n$. Diagonal double excitations are described by diagonal elements $\delta \gamma_{p p}$ and can therefore always be covered. A matrix element $\delta \gamma_{p q}$ indicates contribution from a single excitation $p \rightarrow q$. With the examples treated in the various sections it is demonstrated that the index $p$ in these matrix elements only needs to run over the occupied orbitals, and maybe over one or two orbitals more (the lumo and lumo +1 ) if there are significant off-diagonal double excitations. This effectively reduces the size of the TD1MFT problem to only slightly larger than that of TDDFT. In Sec. VIII the conclusions are drawn.

\section{PINO RESPONSE THEORY}

In TDDFT the central quantity is the time-dependent density, which is treated with the help of effective one-particle equations through the introduction of the KS non-interacting system with the KS orbitals. In the 1RDM related theories the central quantity is the time-dependent 1RDM of Eq. (1), which immediately yields its eigenfunctions, the natural orbitals, as the natural set of one-particle functions to describe its time-development. In a development very much analogous to the one of TDDFT (Ref. 28) we derive effective oneelectron time-dependent Schrödinger type of equations for these orbitals by first considering the action $A$ of the interacting system

$$
A:=\int_{0}^{T} \mathrm{~d} t\left\langle\Psi(t)\left|i \partial_{t}-\hat{H}(t)\right| \Psi(t)\right\rangle,
$$

( $\hat{H}(t)$ is the total time-dependent Hamiltonian) as a functional $A[\{\phi, n\}]$ of the NOs and the NO occupations (ONs). Actually, we have given arguments that one should also introduce into the functional, apart from dependence on the NOs and ONs, a dependence on a set of additional variables, which can conveniently be denoted as the phases of the orbitals. ${ }^{25,26}$ The expansion (1) does not fix the phases of the NOs, i.e., with the NO $\phi_{k}(t)$, the orbital $\varphi_{k}(t)=\phi_{k}(t) \mathrm{e}^{i \beta_{k}(t)}$ is also a NO. Our basic states are therefore not just the NOs, but PINOs. To fix the phases of the orbitals, the stationarity of the action (2) is employed in the PINO theory as a phase-fixing condition. Specifically, the PINO theory postulates that the stationary point of (2)

$$
\delta A[\{\not \not, n\}]=i\langle\Psi(T) \mid \delta \Psi(T)\rangle[\{\not \not, n\}]
$$

is attained with the unique set of PINOs

$$
\pi_{k}(\boldsymbol{x} t)=\phi_{k}(\boldsymbol{x} t) \mathrm{e}^{i \beta_{k}(t)} .
$$

In Eq. (3) the right-hand side is the remainder from the free boundary at time $T .{ }^{29}$ The universal functional $A[\{\not t, n\}]$ is partitioned as follows:

$$
A[\{\not \not, n\}]=A_{0}[\{\not \nexists, n\}]-A_{\mathrm{Hxc}}[\{\not \nexists t, n\}],
$$

where $A_{0}$ is the one-electron part

$$
A_{0}:=\int_{0}^{T} \mathrm{~d} t \sum_{k} n_{k}(t)\left\langle t_{k}(t)\left|i \partial_{t}-\hat{h}(t)\right| \not t_{k}(t)\right\rangle
$$

and $A_{\mathrm{Hxc}}$ is the Hartree-exchange-correlation part dealing with all the two-body effects

$$
\begin{aligned}
A_{\mathrm{Hxc}}[\{\not \not t, n\}]:= & \int_{0}^{T} \mathrm{~d} t\{\langle\Psi(t)|\hat{W}| \Psi(t)\rangle \\
& \left.+i \sum_{k} n_{k}(t)\left\langle\not t_{k}(t) \mid \dot{\not ̆ t}_{k}(t)\right\rangle-i\langle\Psi(t) \mid \Psi(t)\rangle\right\},
\end{aligned}
$$

where $\hat{W}$ is the two-electron part of the Hamiltonian. With the additional orthonormality conditions on the PINOs, Eq. (3) turns to

$$
\begin{aligned}
\delta A_{0} & -\delta \mathcal{W}-\sum_{k l} \lambda_{k l}(t)\left(\left\langle\not t_{k}(t) \mid \delta \pi_{l}(t)\right\rangle+\left\langle\delta \pi_{k}(t) \mid \not t_{l}(t)\right\rangle\right) \\
& =i \sum_{k} n_{k}(T)\left\langle\not t_{k}(T) \mid \delta \mathbb{t}_{k}(T)\right\rangle,
\end{aligned}
$$

where

$$
\begin{aligned}
\delta \mathcal{W}[\{\not t, n\}]:= & \delta A_{\mathrm{Hxc}}+i\langle\Psi(T) \mid \delta \Psi(T)\rangle \\
& -i \sum_{k} n_{k}(T)\left\langle\not_{k}(T) \mid \delta t_{k}(T)\right\rangle .
\end{aligned}
$$

Variation in Eq. (8) with respect to $\mathbb{t}_{k}(\boldsymbol{x} t), \mathbb{t}_{l}^{*}(\boldsymbol{x} t)$, and $n_{k}(t)$ with the subsequent elimination of the Lagrange multipliers $\lambda_{k l}$ from the resultant expressions produces the equation of motion (EOM) for the PINOs (Refs. 25 and 26)

$$
\left(\hat{h}(t)+\hat{v}_{\mathrm{Hxc}}(t)\right) \pi_{k}(\boldsymbol{x} t)=i \partial_{t} \not_{k}(\boldsymbol{x} t)
$$

and the EOM for the PINO occupations

$$
i \dot{n}_{k}(t)=\mathcal{W}_{k k}^{\dagger}(t)-\mathcal{W}_{k k}(t) .
$$

The potential $\hat{v}_{\mathrm{Hxc}}(t)$ in Eq. (10) is defined in terms of its diagonal matrix elements

$$
\left\langle\not \mathbb{t}_{k}(t)\left|\hat{v}_{\mathrm{Hxc}}(t)\right| \not \mathbb{t}_{k}(t)\right\rangle:=\frac{\partial \mathcal{W}(t)}{\partial n_{k}(t)}
$$

and its off-diagonal matrix elements

$$
\left\langle\pi_{k}(t)\left|\hat{v}_{\mathrm{Hxc}}(t)\right| \oiint_{l}(t)\right\rangle:=\frac{\mathcal{W}_{k l}^{\dagger}(t)-\mathcal{W}_{k l}(t)}{n_{l}(t)-n_{k}(t)} \quad \forall_{k \neq l},
$$


where

$$
\mathcal{W}_{k l}(t):=\int \mathrm{d} \boldsymbol{x} \frac{\partial \mathcal{W}}{\partial \mathbb{t}_{k}(\boldsymbol{x} t)} \oiint_{l}(\boldsymbol{x} t) .
$$

In the adiabatic approximation the functional $\mathcal{W}[\{\not t, n\}](t)$ is replaced with the functional $W[\{\not \nexists, n\}]$ of the static electronelectron interaction energy.

We now proceed to derive equations for the excitation energies using the linear response formalism. ${ }^{25,26}$ First consider a time-independent system. The system has stationary solutions for the PINOs of the form

$$
\not{t}_{k}^{0}(\boldsymbol{x} t)=\not_{k}(\boldsymbol{x}) \mathrm{e}^{-i \epsilon_{k} t},
$$

where the time-independent PINOs satisfy

$$
\left(\hat{h}+\hat{v}_{\mathrm{Hxc}}\right) \mathbb{t}_{k}(\boldsymbol{x})=\epsilon_{k} \not_{k}(\boldsymbol{x}) .
$$

Now we apply a time-dependent perturbation to the potential, $\delta \boldsymbol{v}(t)$. The PINOs are now perturbed and we expand the perturbation in them as

$$
\not_{k}(\boldsymbol{x} t)=: \mathrm{e}^{-i \epsilon_{k} t}\left(\mathbb{t}_{k}(\boldsymbol{x})+\delta \boldsymbol{t}_{k}(\boldsymbol{x} t)+\cdots\right) .
$$

The perturbation in the NOs, $\delta t_{k}(x t)$, is expanded in the stationary time-independent PINOs as

$$
\delta \not t_{k}(\boldsymbol{x} t)=: \sum_{r} \not t_{r}(\boldsymbol{x}) \delta U_{r k}(t) .
$$

Since the PINOs are still NOs, the perturbation in the 1RDM is readily expressed as

$$
\delta \gamma_{k l}(t)=\delta n_{k}(t) \delta_{k l}+\left(n_{l}-n_{k}\right) \delta U_{k l}(t) .
$$

Using these expressions for the perturbations, one can derive the frequency dependent response equations. Details of the derivation can be found in Refs. 26 and 27. The results can be summarized as follows. Using the adiabatic approximation, $\mathcal{W} \approx W$, and restricting ourselves to systems with timereversal symmetry, the frequency-dependent response equations can be cast into the following form:

$$
\left(\begin{array}{cc}
\omega \mathbf{1} & -\boldsymbol{A}^{+} \\
-\boldsymbol{D} & \omega \mathbf{1}
\end{array}\right)\left(\begin{array}{l}
\boldsymbol{X}(\omega) \\
\boldsymbol{Y}(\omega)
\end{array}\right)=\left(\begin{array}{c}
\mathbf{0} \\
\boldsymbol{V}(\omega)
\end{array}\right)
$$

The vectors $\boldsymbol{X}$ and $\boldsymbol{Y}$ collect the perturbations in the PINOs and occupation numbers as

$$
\boldsymbol{X}(\omega):=\left(\begin{array}{c}
\delta \boldsymbol{\gamma}^{R}(\omega) \\
\delta \boldsymbol{n}(\omega)
\end{array}\right), \quad \boldsymbol{Y}(\omega):=\left(\begin{array}{c}
i \delta \boldsymbol{U}^{I}(\omega) \\
i \delta \boldsymbol{U}^{D}(\omega) / 2
\end{array}\right),
$$

where the superscript $D$ means only the diagonal elements. The superscripts $R$ and $I$ indicate the Fourier transforms of the real and imaginary parts respectively, i.e.,

$$
f^{R}(\omega):=\mathcal{F}[\mathfrak{R e} f](\omega), \quad f^{I}(\omega):=\mathcal{F}[\mathfrak{I m} f](\omega) .
$$

The superscripts $R$ and $I$ attached to a matrix (here at $\delta \gamma$ and $\delta \boldsymbol{U})$ denote a vector consisting of only the lower off-diagonal matrix elements $\left(\delta \gamma_{k l}^{R}\right.$ and $\left.\delta U_{k l}^{R}, k>l\right)$. Due to the assumed time-reversal symmetry, we only need to consider real PINOs, $t_{k}(\boldsymbol{x})$, so the potential on the right-hand side of Eq. (20) has only real entries

$$
\boldsymbol{V}(\omega):=\left(\begin{array}{c}
\delta \boldsymbol{v}^{R}(\omega) \\
\delta \boldsymbol{v}^{D}(\omega) / 2
\end{array}\right)
$$

The matrix on the left-hand side of Eq. (20) is determined by the unperturbed system and is a rather involved quantity. An important ingredient is the response of the two-electron part in the equations, which are given by the coupling matrices

$$
\begin{aligned}
K_{k l, b a}^{\star t} & :=\int \mathrm{d} \boldsymbol{x}\left(\frac{\partial\left(W_{k l}^{\dagger}-W_{k l}\right)}{\partial \pi_{b}(\boldsymbol{x})} \not_{a}(\boldsymbol{x})-\oiint_{b}^{*}(\boldsymbol{x}) \frac{\partial\left(W_{k l}^{\dagger}-W_{k l}\right)}{\partial \pi_{a}^{*}(\boldsymbol{x})}\right), \\
K_{k l, a}^{n} & :=\frac{\partial\left(W_{k l}^{\dagger}-W_{k l}\right)}{\partial n_{a}}, \\
\bar{W}_{k, a} & :=\frac{\partial^{2} W}{\partial n_{k} \partial n_{a}} .
\end{aligned}
$$

The response of the two-electron part is combined with contributions from the one-electron part

$$
\begin{aligned}
A_{k l, b a} & :=\left(n_{b}-n_{a}\right)\left(h_{k a} \delta_{b l}-\delta_{k a} h_{b l}\right)+K_{k l, b a}^{\hbar}, \\
C_{k l, a} & :=h_{k l}\left(\delta_{a l}-\delta_{k a}\right)+K_{k l, a}^{n} .
\end{aligned}
$$

Now we define the $\boldsymbol{A}^{ \pm}$matrices as

$$
A_{k l, b a}^{ \pm}:=A_{k l, b a} \pm A_{k l, a b} .
$$

The submatrix $\boldsymbol{D}$ is defined as a composition of the previously introduced matrices

$$
\boldsymbol{D}:=\left(\begin{array}{cc}
\boldsymbol{N}^{-1} A^{-} \boldsymbol{N}^{-1} & \boldsymbol{N}^{-1} \boldsymbol{C} \\
\boldsymbol{C}^{T} \boldsymbol{N}^{-1} & \bar{W}
\end{array}\right),
$$

where $N_{k l, b a}:=\left(n_{l}-n_{k}\right) \delta_{k a} \delta_{b l}$. Equation (20) can be manipulated a bit further to reduce the dimensions by eliminating $\boldsymbol{Y}(\omega)$ from the response equations. After some further manipulations we find ${ }^{30}$

$$
\left[\omega^{2} \mathbf{1}-\sqrt{\boldsymbol{A}^{+}} \boldsymbol{D} \sqrt{\boldsymbol{A}^{+}}\right]\left(\boldsymbol{A}^{+}\right)^{-1 / 2} \boldsymbol{X}(\omega)=\sqrt{\boldsymbol{A}^{+}} \boldsymbol{V}(\omega) .
$$

It is clear that at values of $\omega$ which are eigenvalues of the matrix $\sqrt{\boldsymbol{A}^{+}} \boldsymbol{D} \sqrt{\boldsymbol{A}^{+}}$responses may exist even in the absence of a driving field $\boldsymbol{V}(\omega)$. Those eigenvalues must coincide with the "eigenfrequencies" of the system, i.e., its excitation energies. We can also solve for $\boldsymbol{X}(\omega)$ explicitly. Since the matrix on the left-hand side of Eq. (28) is symmetric, it can be inverted. In particular, with the decomposition

$$
\sqrt{\boldsymbol{A}^{+}} \boldsymbol{D} \sqrt{\boldsymbol{A}^{+}} \boldsymbol{F}_{k}=\omega_{k}^{2} \boldsymbol{F}_{k},
$$

we can solve Eq. (28) for $\boldsymbol{X}(\omega)$ as

$$
\boldsymbol{X}(\omega)=\sqrt{\boldsymbol{A}^{+}} \sum_{k} \frac{\boldsymbol{F}_{k} \boldsymbol{F}_{k}^{T}}{\omega^{2}-\omega_{k}^{2}} \sqrt{\boldsymbol{A}^{+}} \boldsymbol{V}(\omega) .
$$

This equation connects the responses collected in the vector $\boldsymbol{X}(\omega)$ with the perturbing field $\boldsymbol{V}(\omega)$, i.e., is of the form $\delta \boldsymbol{\gamma}=\boldsymbol{\chi} \delta \boldsymbol{v}$, where $\chi$ is the 1RDM-1RDM response function. Therefore, comparing Eq. (30) with the Lehmann representation of the 1RDM-1RDM response function will 
enable us to identify the $\omega_{k}$ with the excitation energies and $\sqrt{\boldsymbol{A}^{+}} \boldsymbol{F}_{k} /\left(2 \sqrt{\omega_{k}}\right)$ with the transition 1RDMs, see Sec. III.

\section{CONNECTION OF THE 1RDM RESPONSE VECTOR OF AN EXCITED STATE WITH THE TRANSITION DENSITY MATRIX}

Excited states are characterized by the transition density matrix connecting the ground and excited states. In this section we will establish the relationship between the calculated responses, as embodied in the $\boldsymbol{X}(\omega)$ (or $\boldsymbol{F}_{k}(\omega)$ ), and the transition density matrices. We start with the Lehmann representation of the 1RDM-1RDM response function. Using perturbation theory, ${ }^{31}$ the perturbation in the $1 \mathrm{RDM}, \delta \boldsymbol{\gamma}(t)$, due to a perturbation in the potential, $\delta \boldsymbol{v}(t)$ can be written as

$$
\delta \gamma_{k l}(t)=\int_{-\infty}^{\infty} \mathrm{d} t^{\prime} \sum_{a b} \chi_{k l, b a}\left(t-t^{\prime}\right) \delta v_{a b}\left(t^{\prime}\right) .
$$

The retarded response function, $\chi\left(t-t^{\prime}\right)$, is defined as

$$
\chi_{k l, b a}\left(t-t^{\prime}\right):=-i \theta\left(t-t^{\prime}\right)\left\langle\Psi_{0}\left|\left[\hat{\gamma}_{k l}(t), \hat{\gamma}_{a b}\left(t^{\prime}\right)\right]\right| \Psi_{0}\right\rangle,
$$

where $\hat{\gamma}(t)$ is the 1RDM operator in the Heisenberg picture with elements $\hat{\gamma}_{k l}(t)=\hat{c}_{l}^{\dagger}(t) \hat{c}_{k}(t)$, and the Heaviside step function is defined as

$$
\theta(\tau)= \begin{cases}1 & \text { for } \tau>0 \\ 0 & \text { for } \tau<0\end{cases}
$$

The step function reflects that we have a causal response, i.e., changes in the 1RDM only depend on perturbations in the potential at earlier times. From basic complex analysis, the step function can also be written as

$$
\theta(\tau):=-\lim _{\eta \rightarrow 0^{+}} \int_{-\infty}^{\infty} \frac{\mathrm{d} \omega}{2 \pi i} \frac{\mathrm{e}^{-i \omega \tau}}{\omega+i \eta} .
$$

Inserting a complete set of states and taking the Fourier transform of Eq. (32), we obtain the frequency dependent 1RDM1RDM response function (Lehmann representation)

$\chi(\omega)=\lim _{\eta \rightarrow 0^{+}} \sum_{\alpha}\left(\frac{\Delta \boldsymbol{\gamma}(\alpha) \otimes \Delta \boldsymbol{\gamma}(\alpha)^{\dagger}}{\omega-\Omega_{\alpha}+i \eta}-\frac{\Delta \boldsymbol{\gamma}(\alpha)^{\dagger} \otimes \Delta \boldsymbol{\gamma}(\alpha)}{\omega+\Omega_{\alpha}+i \eta}\right)$,

where $\Omega_{\alpha}$ are the excitation energies and the transition 1 RDMs are defined as

$$
\Delta \gamma_{k l}(\alpha):=\left\langle\Psi_{0}\left|\hat{\gamma}_{k l}\right| \Psi_{\alpha}\right\rangle
$$

and its complex conjugate as

$$
\Delta \gamma_{k l}^{\dagger}(\alpha):=\left\langle\Psi_{0}\left|\hat{\gamma}_{l k}\right| \Psi_{\alpha}\right\rangle^{*}=\left\langle\Psi_{\alpha}\left|\hat{\gamma}_{k l}\right| \Psi_{0}\right\rangle
$$

Note that the in terms in the denominator reflect the causality of the retarded response function, i.e., they come from the Fourier transform of the step function (34), contrary to a common belief which attributes their appearance to some unphysical adiabatic switch-on of the perturbation.

Since our response Eq. (30) only deals with the real part of the 1RDM, we actually only need the $\gamma^{R} \gamma^{R}$ response function

$$
\begin{aligned}
\chi_{k l, b a}^{R R}(\omega)= & \sum_{\alpha} \Delta \gamma_{k l}^{R}(\alpha) \Delta \gamma_{a b}^{R}(\alpha) \\
& \times\left[\mathcal{P} \frac{2 \Omega_{\alpha}}{\omega^{2}-\Omega_{\alpha}^{2}}-i \pi\left(\delta\left(\omega-\Omega_{\alpha}\right)-\delta\left(\omega+\Omega_{\alpha}\right)\right)\right],
\end{aligned}
$$

where $\mathcal{P}$ indicates the Cauchy principal value. Note that we dropped the complex conjugation, since we assumed timereversal symmetry, so the eigenstates of the reference system can be chosen to be real. Since we did not take causality into account in our response Eq. (30), we should only compare the principal value part. The only thing we need to keep in mind is that in Eq. (30) the sum only runs over $a \geq b$, whereas in Eq. (31) the sum runs over all $a, b$. So we need to introduce a factor $1 / 2$ for the off-diagonal elements to make the comparison. Since the diagonal elements of the perturbation already had a factor half in Eq. (23) we can pull it inside the summation of Eq. (30) and find

$$
\begin{aligned}
\mathcal{P} \chi^{R R}(\omega) & =\sum_{\alpha} \Delta \boldsymbol{\gamma}^{R}(\alpha) \otimes \Delta \boldsymbol{\gamma}^{R}(\alpha) \mathcal{P} \frac{2 \Omega_{\alpha}}{\omega^{2}-\Omega_{\alpha}^{2}} \\
& =\sum_{k} \frac{\sqrt{\boldsymbol{A}^{+}} \boldsymbol{F}_{k} \boldsymbol{F}_{k}^{T} \sqrt{\boldsymbol{A}^{+}}}{2\left(\omega^{2}-\omega_{k}^{2}\right)} .
\end{aligned}
$$

Since this relation has to hold for every $\omega$, we find that the eigenvalues $\omega_{k}$ from Eq. (29) are exactly the excitation energies $\Omega_{k}$ and that the real part of the transition 1RDMs are related to the eigevectors $\boldsymbol{F}_{k}$ as

$$
\Delta \boldsymbol{\gamma}^{R}(\alpha)=\frac{\sqrt{\boldsymbol{A}^{+}} \boldsymbol{F}_{\alpha}}{2 \sqrt{\Omega_{\alpha}}}
$$

The imaginary part of the transition $1 \mathrm{RDM}$ is now readily derived, since the perturbations in the real and imaginary part of the 1RDM are related according to Eq. (20). Using the definitions (21), we find

$$
i \Delta \boldsymbol{\gamma}^{I}(\alpha)=\Omega_{\alpha}\left(A^{+}\right)^{-1} \Delta \boldsymbol{\gamma}^{R}(\alpha)=\frac{1}{2} \sqrt{\Omega_{\alpha}} \boldsymbol{N}\left(\boldsymbol{A}^{+}\right)^{-1 / 2} \boldsymbol{F}_{\alpha} .
$$

Another approach to derive this relation would be to write down the $\chi^{I R}(\omega)$ response function and perform a similar analysis as before.

In Sec. IV, Eq. (39) will be used in order to interpret the eigenvectors $\boldsymbol{F}_{k}$ of Eq. (29), to assign the calculated excitations as well as to rationalize the performance of the restricted response calculations.

\section{CONNECTION OF THE TRANSITION DENSITY MATRIX WITH THE “SINGLE-AND-DOUBLE EXCITATION” CHARACTER OF THE EXCITED STATE}

We demonstrate in this section that the TDDMFT response theory is able to represent not only excited states of predominantly singly excited character, but also various types of double excitations. Also charge transfer transitions will be shown to be obtained accurately. At each excitation energy we obtain the corresponding response vector $\boldsymbol{F}_{k}$ and we 
can determine the transition density matrix from Eq. (39). We demonstrate here that the 1RDM responses obtained at the excitation energies afford a representation of transition density matrices for excited states of singly excited and doubly excited character, also from ground states that are not of singlereference type. We assume the wavefunctions of the ground state and excited states are written as linear combinations of determinants based on the NOs as one-electron basis,

$$
\begin{gathered}
\Psi_{0}=C_{0} \Phi_{0}+C_{i}^{a} \Phi_{i}^{a}+C_{i j}^{a b} \Phi_{i j}^{a b}+\ldots, \\
\Psi_{\mathrm{exc}}=D_{0} \Phi_{0}+D_{i}^{a} \Phi_{i}^{a}+D_{i j}^{a b} \Phi_{i j}^{a b}+\ldots,
\end{gathered}
$$

$i, j, k \ldots \leq N$ are indices running over the $N$ (strongly) occupied NOs, $a, b, c \ldots$ run over the virtual (weakly occupied) NOs. Close to the equilibrium geometry this will typically yield a single-reference picture with the determinant $\Phi_{0}$ built from the first $N$ (strongly occupied) NOs as leading term, and small coefficients for the singly, doubly, and more highly excited determinants. We will also consider the case where the ground state has multideterminant character, later in this section. If an excited state is of singly excited $(i \rightarrow a)$ character, the coefficient $D_{0}$ will be small and the $D_{i}^{a}$ coefficient will be large; if it is of doubly excited $(i j \rightarrow a b)$ character, $D_{i j}^{a b}$ will be large, etc. The interpretation of $\Delta \boldsymbol{\gamma}^{R}(\alpha)$ follows immediately by substituting these ground state and excited state wavefunctions in Eq. (36) and using the Slater-Condon rules to evaluate the matrix element. Note that the transition density of Eq. (36) will lead to many determinant-determinant terms,

$$
\begin{aligned}
\Delta \gamma\left(\Phi_{1} \Phi_{2} \mid 1,1^{\prime}\right)= & N \int \Phi_{1}(1,2, \ldots, N) \\
& \times \Phi_{2}^{*}\left(1^{\prime}, 2, \ldots, N\right) \mathrm{d} 2 \cdots \mathrm{d} N .
\end{aligned}
$$

The following rules apply:

- Two equal determinants yield contributions to the diagonal elements $\Delta \gamma_{p p}$ for all $p$ in the determinant

$$
\begin{aligned}
& N \int C_{1} D_{1}^{*} \Phi_{1}(1,2, \ldots, N) \Phi_{1}^{*}\left(1^{\prime}, 2, \ldots, N\right) \mathrm{d} 2 \cdots \mathrm{d} N \\
& \quad=\Sigma_{p=1}^{N} C_{1} D_{1}^{*} \phi_{p}(1) \phi_{p}^{*}\left(1^{\prime}\right) .
\end{aligned}
$$

So all diagonal terms in the product $\Psi_{0} \Psi_{\text {exc }}^{*}$ (i.e., $C_{0} D_{0}^{*} \Phi_{0} \Phi_{0}^{*}, C_{i}^{a} D_{i}^{a *} \Phi_{i}^{a} \Phi_{i}^{a *}, C_{i j}^{a b} D_{i j}^{a b *} \Phi_{i j}^{a b} \Phi_{i j}^{a b *}$, etc. $)$ will contribute to diagonal $\Delta \gamma_{p p}$ elements if they contain the NO $p$.

- Two determinants that differ in one function (e.g., $\Phi_{0}$ and $\Phi_{i}^{a}$ ) yield only one $\Delta \gamma_{p q}$ element, just for the functions in which they differ. For example, for $\Phi_{0}$ and $\Phi_{i}^{a}$,

$$
\begin{aligned}
& N \int C_{0} D_{i}^{a *} \Phi_{0}(1,2, \ldots, N) \Phi_{i}^{a *}\left(1^{\prime}, 2, \ldots, N\right) \\
& \quad \times \mathrm{d} 2, \mathrm{~d} 3 \cdots \mathrm{d} N=C_{0} D_{i}^{a *} \phi_{i}(1) \phi_{a}^{*}\left(1^{\prime}\right) .
\end{aligned}
$$

- Two determinants that differ in two functions yield zero contribution to the $\Delta \boldsymbol{\gamma}$ matrix.
So an $\alpha=(i \rightarrow a)$ singly excited state $\left(D_{i}^{a} \approx 1\right)$ from a single-reference ground state $\left(C_{0} \approx 1\right)$ will lead to a single large $\Delta \boldsymbol{\gamma}^{R}(\alpha)$ matrix element, i.e., $\Delta \gamma_{i a}^{R}(i \rightarrow a) \approx C_{0} D_{i}^{a}$. This will make such a type of excited state easily recognizable. It is to be noted that at zero excitation energy also a solution will be obtained, which corresponds to the ground state. It will be characterized by all diagonal terms $\left(\Delta \gamma_{i i}^{R}\right)(0)$ $\approx\left(C_{0}\right)^{2}, i=1 \ldots N$. An excited state with predominant double excitation character, e.g., $(i j \rightarrow a b)$ (an off-diagonal double, from the closed shells $\left(\phi_{i}\right)^{2}$ and $\left(\phi_{j}\right)^{2}$ to the open shell configuration $\left.\left(\phi_{i}\right)^{1}\left(\phi_{j}\right)^{1}\left(\phi_{a}\right)^{1}\left(\phi_{b}\right)^{1}\right)$, will probably be at high energy if the ground state is of single-determinant type. If we assume that the same doubly excited configuration is the most important one in the ground state wavefunction, this excited state will be characterized with the largest $\Delta \boldsymbol{\gamma}^{R}(\alpha)$ matrix elements for $\Delta \boldsymbol{\gamma}_{a a}^{R}$ and $\Delta \boldsymbol{\gamma}_{b b}^{R}$, as well as for $\Delta \boldsymbol{\gamma}_{k k}^{R}, k \neq i, j$. In the case of a single-reference type ground state, such doubly excited states will usually not be encountered in the interesting (low-energy) part of the excitation spectrum.

It is of interest to consider the case where a single reference is no longer adequate, i.e., a doubly excited determinant, $(i \bar{l} \rightarrow a \bar{a})$, makes an important contribution to the ground state (strong nondynamical correlation). This is of course the case at elongated bond lengths in $\mathrm{H}_{2}$, where a strong mixing of the $\left(1 \sigma_{g}\right)^{2}$ and $\left(1 \sigma_{u}\right)^{2}$ configurations occurs. More generally, we consider the case that $\Psi_{0}$ is approximated with the reference determinant $\Phi_{0}$ built from the $N$ first (strongly occupied) PINOs plus an admixture of a diagonal double excitation $\left(\phi_{i}\right)^{2} \rightarrow\left(\phi_{a}\right)^{2}$, which will usually be from the last strongly occupied NO ( $i=N$, the "homo") to the first weakly occupied NO ( $a=N+1$, the "lumo"),

$$
\Psi_{0} \approx C_{0} \Phi_{0}+C_{h \bar{h}}^{l \bar{l}} \Phi_{h \bar{h}}^{l \bar{l}} .
$$

It can easily be seen that this non-single-reference character of the ground state does not make much difference for singly excited states of $(i \rightarrow a)$ type if $i \neq h$ and $a \neq l$. The matrix element in Eq. (36) between $C_{0} \Phi_{0}$ and $D_{i}^{a} \Phi_{i}^{a}$ will again yield $\Delta \gamma_{i a}^{R}=C_{0} D_{i}^{a}$. If the $\left(\phi_{h}\right)^{2} \rightarrow\left(\phi_{l}\right)^{2}$ determinant also makes a large contribution to the $(i \rightarrow a)$ singly excited state, i.e., the contribution of the configuration with simultaneously $(i \rightarrow a)$ and $\left(\phi_{h}\right)^{2} \rightarrow\left(\phi_{l}\right)^{2}$ is large in $\Psi_{\mathrm{exc}}$, then also a significant contribution $\Delta \gamma_{i a}^{R}=C_{h \bar{h}}^{l \bar{l}} D_{h \bar{h}, i}^{l l, a}$ will arise,

$$
\Delta \gamma_{i a}^{R}\left(\Psi_{0}(45) \rightarrow \Psi_{\mathrm{exc}}(i \rightarrow a)\right)=C_{0} D_{i}^{a}+C_{h \bar{h}}^{l \bar{l}} D_{h \bar{h}, i}^{l \bar{l}, a} .
$$

The special single excitation $(h \rightarrow l)$ between the two orbitals involved in the nondynamical correlation embodied in wavefunction (45), with $\Psi_{\mathrm{exc}} \approx D_{h}^{l} \Phi_{h}^{l}, D_{h}^{l} \approx 1$, will have a matrix element (36) of $\Psi_{\text {exc }}$ with both $\Phi_{0}$ and $\Phi_{h \bar{h}}^{l i}$, so

$$
\Delta \gamma_{h l}^{R}\left(\Psi_{0}(45) \rightarrow \Psi_{\mathrm{exc}}(h \rightarrow l)\right)=C_{0} D_{h}^{l}+C_{h \bar{h}}^{l \bar{l}} D_{h}^{l} .
$$

So all excited states of single excitation nature are characterized by a single large transition density matrix element $\Delta \gamma_{i a}^{R}$ (including $\Delta \gamma_{h l}^{R}$ ), with corresponding large response vector element.

Of special interest is a possible "double excitation" structure in the $1 \mathrm{RDM}$ response. In the case of the ground state 
(45), the excitation to the "diagonal doubly excited" state $\left(\phi_{h}\right)^{2} \rightarrow\left(\phi_{l}\right)^{2}$ with $\Psi_{\mathrm{exc}} \approx D_{0} \Phi_{0}+D_{h \bar{h}}^{l \bar{l}} \Phi_{h \bar{h}}^{l \bar{l}}=-C_{h \bar{h}}^{l \bar{l}} \Phi_{0}$ $+C_{0} \Phi_{h \bar{h}}^{l \bar{l}}$ can be important. This excitation will lead to large diagonal elements

$$
\Delta \gamma_{h h}^{R}=\Delta n_{h} \approx-C_{0} C_{h \bar{h}}^{l \bar{l}}, \quad \Delta \gamma_{l l}^{R}=\Delta n_{l} \approx C_{h \bar{h}}^{l \bar{l}} C_{0},
$$

(note that the contributions to all other $\Delta \gamma_{i i}^{R}, i \neq h$, arising according to Eq. (43) from the $\left\langle\Phi_{0} \mid \Phi_{0}\right\rangle_{2 \ldots N}$ and $\left\langle\Phi_{h \bar{h}}^{l \bar{l}} \mid \Phi_{h \bar{h}}^{l \bar{l}}\right\rangle_{2 \ldots N}$ integrals, cancel). One obtains $\Delta n_{h}=1 / 2$ and $\Delta n_{l}=-1 / 2$ in the case of equal mixing of the two determinants $\left(C_{0}=-C_{h \bar{h}}^{l \bar{l}}=1 / \sqrt{2}\right)$. The ground state will then be characterized by $\Delta n_{h}=\Delta n_{l}=+1 / 2$. For the ground state wavefunction (45) embodying nondynamical correlation, offdiagonal double excitations will be important if they involve $\phi_{h}$ and $\phi_{l}$. One possibility is excitation of the two electrons in $\left(\phi_{h}\right)^{2}$ to $\left(\phi_{l}\right)^{1}\left(\phi_{a}\right)^{1}$. The other possibility is an excitation $\phi_{i} \phi_{h}$ to $\left(\phi_{l}\right)^{2}$. A configuration $\left(\phi_{l}\right)^{1}\left(\phi_{a}\right)^{1}$ will have zero contribution to the transition density matrix from the $\Phi_{0}$ component of the ground state, but it has a nonzero contribution from the matrix element with $\Phi_{h \bar{h}}^{\bar{l}}$, being of single-excitation type with respect to that determinant. The corresponding transition density matrix element will then be

$$
\Delta \gamma_{l a}^{R}\left(\Psi_{0}(45) \rightarrow \Psi_{\mathrm{exc}}\left(h^{2} \rightarrow l a\right)\right)=C_{h \bar{h}}^{l \bar{l}} D_{h \bar{h}}^{l a} .
$$

which is significant if the configuration $\left(\phi_{h}\right)^{0}\left(\phi_{l}\right)^{2}$ makes an important contribution to the ground state $\left(C_{h \bar{h}}^{l \bar{l}} \gg 0\right)$. Instead of describing this as an off-diagonal double excitation with respect to $\Phi_{0}$ we note that a large $\Delta \gamma_{l a}^{R}$ apparently describes a single excitation from the lumo to a higher orbital, which can clearly only be important in the nondynamical correlation case of wavefunction (45), where the lumo becomes occupied. It is covered in the $\Delta \gamma^{R}$ response vector by extending the range of the $i$ index beyond the homo to include also the lumo. The configuration $\phi_{i} \phi_{h}$ to $\left(\phi_{l}\right)^{2}$ may in the same way be described as a single excitation $\phi_{i} \rightarrow \phi_{h}$ with respect to the determinant $\Phi_{h \bar{h}}^{l \bar{l}}$, becoming important when this determinant has significant weight in the ground state wavefunction, i.e., when $C_{h \bar{h}}^{l \bar{l}} \gg 0$. In that case the transition density matrix element becomes

$$
\Delta \gamma_{i h}^{R}\left(\Psi_{0}(45) \rightarrow \Psi_{\text {exc }}\left(i h \rightarrow l^{2}\right)\right)=C_{h \bar{h}}^{l \bar{l}} D_{i h}^{l \bar{l}} .
$$

The special cases of Eqs. (49) and (50) demonstrate the meaning that can be given to these "virtual $\rightarrow$ virtual" and "occupied $\rightarrow$ occupied" type of excitations.

The qualitative analysis of this section shows that, unlike the density response in TDDFT, which is limited to a response vector with only occupied-virtual ( $i a)$ elements, giving it an essentially "single-excitation" structure, the 1RDM response displays "single-and-double" excitation structure. Furthermore, the present analysis suggests an efficient strategy for the $1 \mathrm{RDM}$ response calculations. One can severely restrict the size of the response calculations retaining only those elements which will attain significant values. We always retain all diagonal elements (occupation number responses $\Delta n_{k}$ ). This will incorporate possible nondynamical correlation effects as embodied in wavefunction (45) and the double excitations characterized by the occupation number changes of Eq. (48). A first restriction would include only "occupied- all" elements $\Delta \gamma_{i, a}$, where $i$ runs to $h$ and $a$ runs over the full PINO set. This restricted variant, denoted R0, is roughly equivalent in size to the response TDDFT. In a slightly extended variant, denoted $\mathrm{R} 1$, the elements of the $\mathrm{R} 0$ variant are augmented with the elements $\Delta \gamma_{l a}$, i.e., the homo+1 (lumo) is included in the range of index $i$. In a further extension we use variant R2, in which the next higher orbital (homo+2) is also included in the range of the first index. We have in this paper not studied any restriction of $i$ within the occupied set or of $a$ within the virtual set, since we have only one occupied orbital. Obviously, in a many-electron system it will be interesting to test restriction of the range of the indices also in the set of occupied and virtual PINOs.

The established "single-and-double" excitation structure of the 1RDM response suggests that time-dependent PINO functional theory could produce single and double excitations already in the adiabatic approximation. This will be demonstrated in Secs. V-VII. Of course, we require an accurate functional which captures the dynamics of the occupation numbers. For singlet two-electron systems, we actually know the exact PINO functional. This is the Löwdin-Shull expression for the total energy of a two-electron system, in which use is made of the finding that the wavefunction in NO basis can be written as a summation over diagonal doubly excited determinants, with the square roots of the occupation numbers as coefficients, each coefficient having positive or negative phase. This energy expression thus depends not only on the NOs and the NO occupation numbers, but also on the additional phase factors which we can introduce as phases of the PINOs. We denote the PINO energy expression obtained in this way as the phase-including Löwdin-Shull functional, PILS

$$
W^{\mathrm{PILS}}=\frac{1}{2} \sum_{k l} \sqrt{n_{k} n_{l}} w_{k k l l}
$$

where

$$
w_{k l b a} \equiv \int \mathrm{d} \boldsymbol{x} \int \mathrm{d} \boldsymbol{x}^{\prime} \nexists_{k}^{*}(\boldsymbol{x}) \not t_{l}^{*}\left(\boldsymbol{x}^{\prime}\right) w\left(\boldsymbol{x}, \boldsymbol{x}^{\prime}\right) \not t_{b}\left(\boldsymbol{x}^{\prime}\right) \not t_{a}(\boldsymbol{x}) .
$$

In ground state calculations similar $\sqrt{n}$-based functionals have been used, but with exchange like integrals $w_{k l l k}$ instead of the $w_{k k l l}$ of Eq. (52). This is the natural choice in the Müller derivation ${ }^{32}$ of the $\sqrt{n}$ functional since it is based on extension of the exchange energy expression. It is one of the two choices in the Buijse-Baerends derivation ${ }^{33,34}$ of this functional. With this phase choice the functional is a genuine density matrix functional, not depending on the phase of the NOs. If the orbitals are all real, this does not make any difference in the ground state total energy. However, the phase choice does play a role in the response calculations. The functional with the $w_{k l k}$ phase choice, denoted the density-matrix adaptation of the Löwdin-Shull energy (DMLS functional), indeed leads to complications in response calculations. ${ }^{25,26}$ In fact, the seemingly innocuous exchange-like phase choice in this functional leads to disastrous response results if the calculations are pushed to higher accuracy than R2. ${ }^{27}$ In Secs. V-VII, we will concentrate on the PILS functional for calculations of the excitation energies in the restricted R0, R1 and $\mathrm{R} 2$ variants. 


\section{APPLICATIONS: POTENTIAL ENERGY CURVES FOR THE ${ }^{1} \Sigma_{u}^{+}$EXCITED STATES OF $\mathrm{H}_{2}$}

In this section the results of comparative calculations of the potential energy curves for the lowest excited states of the ${ }^{1} \Sigma_{u}^{+}$symmetry of the $\mathrm{H}_{2}$ molecule are presented. The potential curves for the lowest four ${ }^{1} \Sigma_{u}^{+}$states are displayed in Fig. 1. The reference full configuration interaction (FCI) calculations have been performed in the aug-cc-pVTZ basis ${ }^{35}$ with the DALTON package. ${ }^{36}$

The ground state of $\mathrm{H}_{2}$ is characterized with the twoelectron bond $\mathrm{H}-\mathrm{H}$. It can be roughly described with the expansion (45) where the coefficient $C_{0}$ of the configuration $\left(1 \sigma_{g}\right)^{2}$ decreases and the (absolute value of the) coefficient $C_{1 \sigma_{g} 1 \sigma_{g}}^{1 \sigma_{g} 1 \sigma_{u}}$ of the configuration $\left(1 \sigma_{u}\right)^{2}$ increases with $R(\mathrm{H}-\mathrm{H})$. The lowest ${ }^{1} \Sigma_{u}^{+}$state $\left(B^{1} \Sigma_{u}^{+}\right)$goes at long distance to an ionic configuration, $\mathrm{H}_{a}^{+}-\mathrm{H}_{b}^{-}-\mathrm{H}_{a}^{-}-\mathrm{H}_{b}^{+}$, with high energy because of the repulsion between two electrons, which are instantaneously located on the same $\mathrm{H}$ atom (in the asymptotic limit of $R \rightarrow \infty$ the lowest ${ }^{1} \Sigma_{u}^{+}$goes to a covalent state with one $\mathrm{H}$ atom in the $1 s \rightarrow 2 s$ excited state ${ }^{37}$ ). Near the equilibrium $\mathrm{H}-\mathrm{H}$ distance, all four ${ }^{1} \Sigma_{u}^{+}$states are, predominantly, single excitations from the $1 \sigma_{g}$ orbital to orbitals of $\sigma_{u}$ type. In the representation of the HF orbitals, the $1^{1} \Sigma_{u}^{+}$

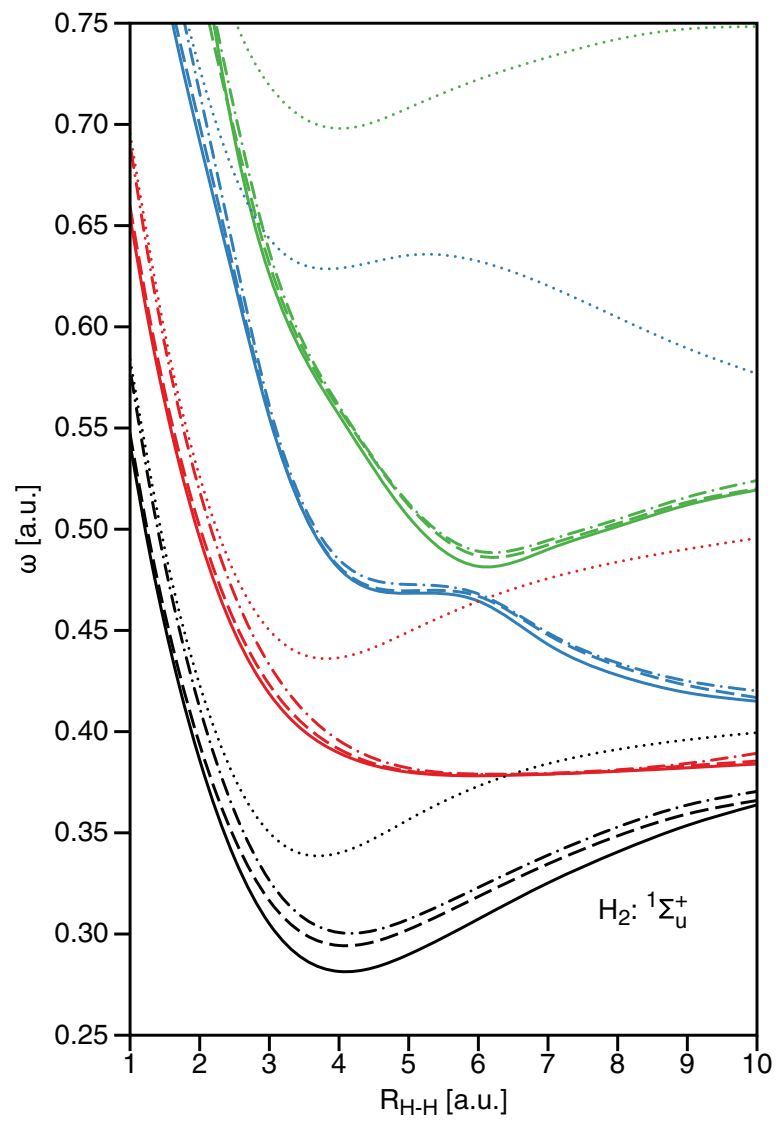

FIG. 1. $\mathrm{H}_{2}{ }^{1} \Sigma_{u}^{+}$excitation energies along the bond breaking coordinate calculated exactly (full CI) and with PINO response calculations with the PILS functional. Full CI: solid lines; PILS-R0: dots; PILS-R1: dotted-dash; PILSR2: dashes. Black, red, blue, green for 1st, 2nd, 3rd, and 4th excited states, respectively. and $2^{1} \Sigma_{u}^{+}$states are, mainly, combinations of the configurations $\left(1 \sigma_{g}\right)^{1}\left(1 \sigma_{u}\right)^{1}$ and $\left(1 \sigma_{g}\right)^{1}\left(2 \sigma_{u}\right)^{1}$, while the $3^{1} \Sigma_{u}^{+}$ and $4^{1} \Sigma_{u}^{+}$states correspond, at equilibrium distance, to the configurations $\left(1 \sigma_{g}\right)^{1}\left(3 \sigma_{u}\right)^{1}$ and $\left(1 \sigma_{g}\right)^{1}\left(4 \sigma_{u}\right)^{1}$, respectively. We are describing the composition of the excited states in terms of the familiar HF orbital based configurations. The NOs are numbered by decreasing occupation number, and NOs with high number may be important in low-lying excited states, see, e.g., Table IV of Ref. 4. The composition of the NOs in terms of the HF orbitals can be used to make the necessary identification of the type of excited state. Here we do this directly with the HF orbitals since the CI on HF orbital basis is available. Note however that the crucial $1 \sigma_{u}$ orbital, involved in the static correlation in $\mathrm{H}_{2}$ at stretched bond lengths, has the largest occupation of the virtual orbitals and is also the "lumo" (orbital $h+1$ ) in the NO basis.

Upon bond stretching there is increasing doubleexcitation character (to the $\left(1 \sigma_{u}\right)^{2}$ configuration) in the ground state $1^{1} \Sigma_{g}^{+}$(see Sec. VI on the ${ }^{1} \Sigma_{g}^{+}$states) which leads to changes in the composition of the excited states. For instance, while in the $\Sigma_{u}^{+}$symmetry, around $R(\mathrm{H}-\mathrm{H})=5$ a.u. the configuration of the lowest excited $1^{1} \Sigma_{u}^{+}$state is $\left(1 \sigma_{g}\right)^{1}\left(1 \sigma_{u}\right)^{1}$ also at elongated distances, the next states, $2^{1} \Sigma_{u}^{+}$and $3^{1} \Sigma_{u}^{+}$, obtain at that distance strong admixtures of (off-diagonal) doubly excited configurations. The $2^{1} \Sigma_{u}^{+}$ retains $50 \%\left(1 \sigma_{g}\right)^{1}\left(2 \sigma_{u}\right)^{1}$ and acquires $31 \%$ of the offdiagonal doubly excited $\left(1 \sigma_{u}\right)^{1}\left(2 \sigma_{g}\right)^{1}$. The $3^{1} \Sigma_{u}^{+}$acquires $40 \%$ of the single excitation $\left(1 \sigma_{g}\right)^{1}\left(2 \sigma_{u}\right)^{1}$ and $37 \%$ of the offdiagonal double excitation $\left(1 \sigma_{u}\right)^{1}\left(3 \sigma_{g}\right)^{1}$. The $4^{1} \Sigma_{u}^{+}$excited state becomes even predominantly doubly excited, namely, a mixture of the off-diagonal doubly excited configurations $\left(1 \sigma_{u}\right)^{1}\left(3 \sigma_{g}\right)^{1}(48 \%)$ and $\left(1 \sigma_{u}\right)^{1}\left(2 \sigma_{g}\right)^{1}(14 \%)$. Note that in all these cases the off-diagonal doubly excited configurations may also be interpreted as single excitations from the $\left(1 \sigma_{u}\right)^{2}$ configuration which contributes strongly to the ground state because of the nondynamical correlation.

The results of the calculations for the lowest four ${ }^{1} \Sigma_{u}^{+}$states are shown in Fig. 1. The TDDFT calculation (not shown in the figure) for the lowest ${ }^{1} \Sigma_{u}^{+}$state shows the familiar TDDFT failure ${ }^{6,7}$ of going steeply to zero at long $R$. In this case the TDDFT zero-order estimate $\omega_{\alpha}^{s}=\epsilon\left(1 \sigma_{u}\right)-\epsilon\left(1 \sigma_{g}\right)$, the difference between the energies of the antibonding and bonding $\mathrm{KS}$ orbitals, vanishes with $R(\mathrm{H}-\mathrm{H})$. In order to compensate this vanishing, the $\mathrm{xc}$ kernel $f_{\mathrm{xc}}$ of TDDFT should diverge. ${ }^{6}$ However, the functions $f_{\mathrm{xc}}$ of ALDA and AGGAs remain finite in this case. As a result, the TDDFT excitation energy vanishes for larger bond distances and the corresponding potential curve does not exhibit a minimum (see Fig. 1 and Ref. 7).

The novel features of Fig. 1 are the potential curves obtained with the restricted response calculations (see Sec. IV) with the PILS functional. The calculations are performed in the same aug-cc-pVTZ basis ${ }^{35}$ as the reference FCI ones. For each excited state the dots show the curve obtained with the response vector restricted to occ. $\rightarrow$ all $\Delta \gamma_{i a}^{R}$ elements, $i \leq$ $N, a \in$ all (the $\Delta \gamma_{p p}^{R}=\Delta n_{p}$, all $p$, are always included). These are calculations of TDDFT like size of the secular 
problem, which we denote R0. Unlike TDDFT, the PINO response calculations properly describe the ionic nature of the $1^{1} \Sigma_{u}^{+}$and $2^{1} \Sigma_{u}^{+}$states of $\mathrm{H}_{2}$ at the distance regime in Fig. 1, producing finite and sizable excitation energies at larger $R(\mathrm{H}-\mathrm{H})$. The TDDFT catastrophe of zero excitation energies at large $R$ has disappeared. It is remarkable that the higher excited states (already $2^{1} \Sigma_{u}^{+}$but notably $3^{1} \Sigma_{u}^{+}$ and $4^{1} \Sigma_{u}^{+}$) show much poorer performance for the R0 type calculations. This is a simple consequence of the fact that those excited states have large doubly excited character, to the $\left(1 \sigma_{u}\right)^{1}\left(2 \sigma_{g}\right)^{1}$ for the $2^{1} \Sigma_{u}^{+}$, to the $\left(1 \sigma_{u}\right)^{1}\left(3 \sigma_{g}\right)^{1}$ configuration for $3^{1} \Sigma_{u}^{+}$and to $\left(1 \sigma_{u}\right)^{1}\left(3 \sigma_{g}\right)^{1}$ and $\left(1 \sigma_{u}\right)^{1}\left(2 \sigma_{g}\right)^{1}$ for $4^{1} \Sigma_{u}^{+}$. For the same reason the TDDFT curves for these states are very poor. ${ }^{3}$ Just going to the R1 variant, with only the $1 \sigma_{u}$ "lumo" included in the range for the $i$ index, as expected completely remedies the deficiency of the R0 type calculation, as is evident from the dot-dashed curves in Fig. 1. Further, but much less striking, improvement is obtained by adding one more orbital to the "occupied" range (R2 variant, dashes). It is clear that the primary improvement afforded by the TDDMFT method comes from the possibility to incorporate the effect of the strong nondynamical correlation by allowing excitations out of the $\left(1 \sigma_{u}\right)^{2}$ configuration which participates strongly in the ground state. The need for such an extension can be observed immediately from the abnormally high occupation number (for a "virtual" orbital) of this orbital when the nondynamical correlation is strong.

At shorter distances, one can notice a very good performance of the PINO response calculations in all $\mathrm{R}$ variants. Already PILS-R0 produces reasonable $\omega_{\alpha}$ at shorter separations $R(\mathrm{H}-\mathrm{H}) \leq 3$ a.u. This is understandable, since this basic variant includes single excitations, which are dominant at those distances. They are markedly improved over the TDDFT calculations. In particular the higher excited states give poor TDDFT results (see Ref. 3), whereas the R0 results are already very good. In the R2 variant the results become extremely accurate for all states except $1^{1} \Sigma_{u}^{+}$.

To provide a more quantitative information on the excitation energies in "normal" single reference situations, Table I displays $\omega_{\alpha}$ values calculated for the equilibrium bond distance $R(\mathrm{H}-\mathrm{H})=1.401$ a.u. for the $\mathrm{R} 0, \mathrm{R} 1$, and $\mathrm{R} 2$ variants. In the R2 variant the elements of the R1 variant are further augmented with the elements $\delta \gamma_{3, a}$ between the third $($ homo+2) and higher NOs. One can see from Table I that there is a consistent overestimation of the excitation energies in the restricted PILS calculations, which gradually decreases when going from PILS-R0 to PILS-R1 to PILS-R2. An interesting observation is that each restricted variant is characterized with its own, nearly monotonic upward shift of all

TABLE I. Excitation energies (in hartree) for 4 lowest ${ }^{1} \Sigma_{u}^{+}$states of the $\mathrm{H}_{2}$ molecule at the equilibrium bond distance.

\begin{tabular}{lcccc}
\hline \hline State & $1^{1} \Sigma_{u}^{+}$ & $2^{1} \Sigma_{u}^{+}$ & $3^{1} \Sigma_{u}^{+}$ & $4^{1} \Sigma_{u}^{+}$ \\
\hline PILS-R0 & 0.506 & 0.614 & 0.816 & 0.920 \\
PILS-R1 & 0.499 & 0.608 & 0.814 & 0.911 \\
PILS-R2 & 0.474 & 0.584 & 0.789 & 0.890 \\
FCI & 0.468 & 0.578 & 0.784 & 0.882 \\
\hline \hline
\end{tabular}

excitation energies from the reference CI ones. Indeed, the PILS-R0 energies exhibit the largest such shift of $\sim 0.04$ a.u. This shift is reduced to $\sim 0.03$ a.u. for the PILS-R1 energies. Further extension from R1 to R2 removes the largest part of the remaining discrepancy and the PILS-R2 energies reproduce rather quantitatively the FCI reference with the upward shift of only 0.006 a.u. (see Table I).

We finally note that the DMLS functional (no results shown), mentioned at the end of Sec. IV, in spite of being a proper 1RDM functional with invariance with respect to the NO phases, is prone in some regions to an erratic description of excited states with our limited (R0-R2) schemes. The deficiency of the DMLS functional becomes much more pronounced when the calculations are further extended. Upon extending the range of the $i$ index to all basis functions, the calculated spectrum exhibits very many spurious excitations that behave erratically as a function of the distance. ${ }^{27}$

\section{APPLICATIONS: POTENTIAL ENERGY CURVES FOR THE ${ }^{1} \Sigma_{g}^{+}$EXCITED STATES OF $\mathrm{H}_{2}$}

In this section, we proceed with the $\mathrm{H}_{2}$ potential energy curves for the lowest states of the ${ }^{1} \Sigma_{g}^{+}$symmetry displayed in Fig. 2. Just as the ${ }^{1} \Sigma_{u}^{+}$states discussed in Sec. V, the lowest ${ }^{1} \Sigma_{g}^{+}$excited states have single-excitation character near the equilibrium distance. In the representation of the HF orbitals, the $n^{1} \Sigma_{g}^{+}$states, $n=2 \ldots 5$, of Fig. 2 correspond to the single-electron promotions $1 \sigma_{g} \rightarrow n \sigma_{g}$ from the $1 \sigma_{g}$ orbital to the higher $n \sigma_{g}$ Rydberg-like orbital of the same symmetry. The $5^{1} \Sigma_{g}^{+}$state has mixed character at $R_{e}$, the largest contributions being $27 \%$ and $19 \%$ from the $\left(2 \sigma_{u}\right)^{2}$ and $\left(1 \sigma_{g}\right)^{1}\left(5 \sigma_{g}\right)^{1}$ configurations, respectively.

With the bond stretching, the single excitation $1 \sigma_{g}$ $\rightarrow 2 \sigma_{g}$ character is transferred from the lowest excited state,

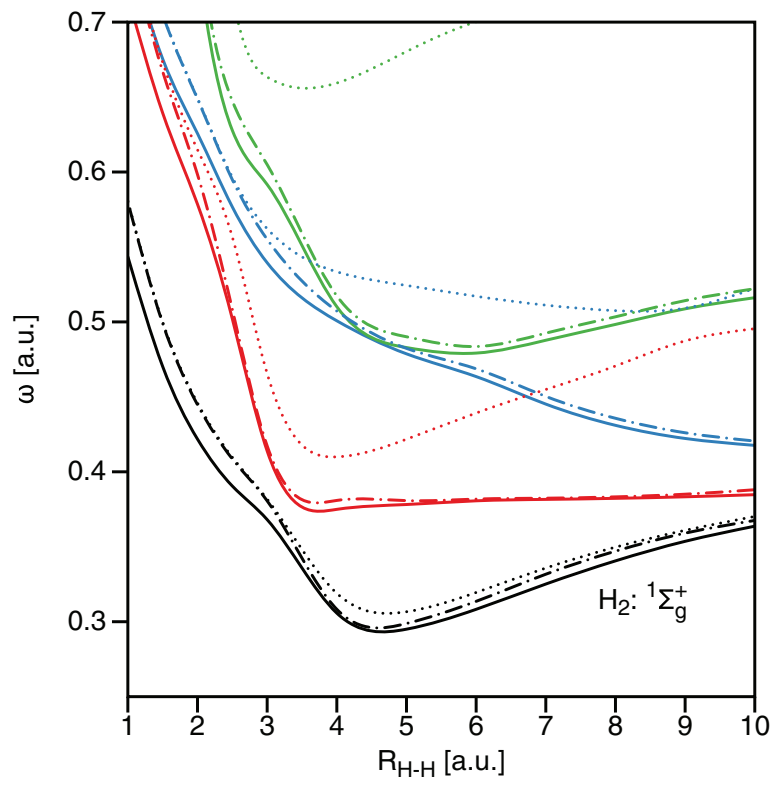

FIG. 2. Exact and PILS calculations of $\mathrm{H}_{2}{ }^{1} \Sigma_{g}^{+}$excitation energies along the bond breaking coordinate. Full CI: solid lines; PILS-R0: dots; PILS-R1: dotted-dashed lines. Black, red, blue, green: $2^{1} \Sigma_{g}^{+}, 3^{1} \Sigma_{g}^{+}, 4^{1} \Sigma_{g}^{+}$, and $5^{1} \Sigma_{g}^{+}$ excited states, respectively. 
$2^{1} \Sigma_{g}^{+}\left(E F^{1} \Sigma_{g}^{+}\right.$in spectroscopic notation) to the next state, $3^{1} \Sigma_{g}^{+}\left(H \bar{H}^{1} \Sigma_{g}^{+}\right)$, through their avoided crossing at $R(\mathrm{H}-\mathrm{H})$ around 3 a.u. (see Fig. 2). The lowest excited state, $2^{1} \Sigma_{g}^{+}$, then acquires double-excitation character to $\left(1 \sigma_{u}\right)^{2}$. This double excitation character is at much higher energies at shorter bond distances, see Fig. 2(b) of Ref. 3. Around 5 a.u. $2^{1} \Sigma_{g}^{+}$ becomes mainly the plus combination of the ground state configuration $\left(1 \sigma_{g}\right)^{2}$ and the diagonal double excitation $\left(1 \sigma_{u}\right)^{2}$, with comparable weights. It is elementary to show that this plus combination leads to ionic nature of the $2^{1} \Sigma_{g}^{+}$state (just as the minus combination gives the covalent Heitler-London configuration in the ground state, $1^{1} \Sigma_{g}^{+}$). Asymptotically, the $2^{1} \Sigma_{g}^{+}$state therefore tends to similar energy as the similarly ionic $2^{1} \Sigma_{u}^{+}$state in Fig. 1, at least in the distance regime shown in the plots (note that at longer distance the $2^{1} \Sigma_{g}^{+}$and $1^{1} \Sigma_{u}^{+}$become covalent again, going to symmetry combinations of $\mathrm{H}(1 s) \mathrm{H}(2 s, 2 p)$ atomic states. ${ }^{37}$

After the avoided crossing of the next two states, $3^{1} \Sigma_{g}^{+}$ and $4{ }^{1} \Sigma_{g}^{+}$, near $R(\mathrm{H}-\mathrm{H})=4.5$ a.u. (see Fig. 2 ), the lower $3{ }^{1} \Sigma_{g}^{+}$ state has at 5 bohr $62 \%$ of the singly excited $\left(1 \sigma_{g}\right)^{1}\left(2 \sigma_{g}\right)^{1}$ configuration and acquires $12 \%$ of the diagonal $\left(1 \sigma_{u}\right)^{2}$ and $7 \%$ of the off-diagonal $\left(1 \sigma_{u}\right)^{1}\left(2 \sigma_{u}\right)^{1}$ doubly excited configuration. It will ultimately tend to one of the covalent states with one atom in a $1 s \rightarrow 2 s, 2 p$ excited state, the other one in the ground state. The higher $4^{1} \Sigma_{g}^{+}$state acquires $46 \%$ of the off-diagonal doubly excited $\left(1 \sigma_{u}\right)^{1}\left(2 \sigma_{u}\right)^{1}$ configuration, in addition to $25 \%$ of the singly excited $\left(1 \sigma_{g}\right)^{1}\left(4 \sigma_{g}\right)^{1}$ configuration. The $5^{1} \Sigma_{g}^{+}$state has at 5 bohr $72 \%$ of the singly excited $\left(1 \sigma_{g}\right)^{1}\left(3 \sigma_{g}\right)^{1}$ configuration and acquires $7 \%$ of the diagonal $\left(1 \sigma_{u}\right)^{2}$ and $6 \%$ of the off-diagonal $\left(1 \sigma_{u}\right)^{1}\left(3 \sigma_{u}\right)^{1}$ doubly excited configurations. Note, that the $2^{1} \Sigma_{g}^{+}$state of the stretched $\mathrm{H}_{2}$ is a striking example of excited states, which are missed in adiabatic TDDFT. ${ }^{3}$ It is instructive to realize, that even the lowest excited state of this prototype two-electron bond molecule is missed in standard TDDFT, because it has doubleexcitation character. The underlying reason of this TDDFT failure is the single-excitation structure of the TDDFT density response discussed in the Introduction.

It is an important virtue of the present adiabatic PINO theory, that it reproduces the entire $2^{1} \Sigma_{g}^{+}$potential energy curve along with those of other low-lying doubly excited states. Already, the basic PILS-R0 reproduces reasonably well the reference FCI curve for the lowest $2^{1} \Sigma_{g}^{+}$excited state, $2^{1} \Sigma_{g}^{+}$, and PILS-R1 further improves the calculated excitation energy, especially, in the interval of 4-5.5 a.u. (see Fig. 2). It is striking that the "TDDFT-like" R0 variant is still rather bad for the $3^{1} \Sigma_{g}^{+}, 4^{1} \Sigma_{g}^{+}$and particularly $5^{1} \Sigma_{g}^{+}$ states, although this poor behavior is immediately remedied if one goes to the only slightly more expensive R1 variant. This behavior is again easily explained from the compositions of the excited states. At longer distances the higher excited states $3^{1} \Sigma_{g}^{+}-5^{1} \Sigma_{g}^{+}$acquire increasing doubly excited character. The $3^{1} \Sigma_{g}^{+}$state has at 5 a.u. $62 \%$ of the singly excited $\left(1 \sigma_{g}\right)^{1}\left(2 \sigma_{g}\right)^{1}$ configuration and acquires $12 \%$ of the diagonal $\left(1 \sigma_{u}\right)^{2}$ and $7 \%$ of the off-diagonal $\left(1 \sigma_{u}\right)^{1}\left(2 \sigma_{u}\right)^{1}$ doubly excited configurations. The $4^{1} \Sigma_{g}^{+}$state retains $25 \%$ of the singly excited $\left(1 \sigma_{g}\right)^{1}\left(4 \sigma_{g}\right)^{1}$ configuration and acquires $46 \%$ of the
TABLE II. Excitation energies (in hartree) for 4 lowest ${ }^{1} \Sigma_{g}^{+}$states of the $\mathrm{H}_{2}$ molecule at the equilibrium bond distance, using the restricted variants (R0-R2) of the PINO response calculations.

\begin{tabular}{lcccc}
\hline \hline State & $2^{1} \Sigma_{g}^{+}$ & $3{ }^{1} \Sigma_{g}^{+}$ & $4{ }^{1} \Sigma_{g}^{+}$ & $5^{1} \Sigma_{g}^{+}$ \\
\hline PILS-R0 & 0.513 & 0.687 & 0.719 & 1.157 \\
PILS-R1 & 0.513 & 0.682 & 0.719 & 1.071 \\
PILS-R2 & 0.486 & 0.656 & 0.694 & 1.064 \\
FCI & 0.483 & 0.653 & 0.688 & 1.039 \\
\hline \hline
\end{tabular}

off-diagonal doubly excited $\left(1 \sigma_{u}\right)^{1}\left(2 \sigma_{u}\right)^{1}$ configuration. The $5^{1} \Sigma_{g}^{+}$state retains $72 \%$ of the singly excited $\left(1 \sigma_{g}\right)^{1}\left(3 \sigma_{g}\right)^{1}$ configuration and acquires $7 \%$ of the diagonal $\left(1 \sigma_{u}\right)^{2}$ and $6 \%$ of the off-diagonal $\left(1 \sigma_{u}\right)^{1}\left(3 \sigma_{u}\right)^{1}$ doubly excited configurations. We note from these compositions that the R1 variant, allowing the configurations with one occupied $1 \sigma_{u}$ orbital ("single excitation out of $1 \sigma_{u}$ ") plus of course the diagonal doubles, should perform well, and indeed it does. We note that the TDDFT calculations for these higher excited states go totally astray, see Fig. 2 in Ref. 4, in agreement with the (diagonal and off-diagonal) double excitation character of these states.

In Table II the excitation energies of the ${ }^{1} \Sigma_{g}^{+}$states calculated at the equilibrium bond distance are presented. Again, the restricted PILS calculation consistently overestimate $\omega_{\alpha}$. Note, that PILS-R1 has nearly the same almost uniform upward shift of 0.03 a.u. of its energies as for the ${ }^{1} \Sigma_{u}^{+}$states of Sec. V. PILS-R0 produces, basically, the same energies of the first three excited states as PILS-R1, while the error of the calculated $5^{1} \Sigma_{g}^{+}$state appreciably decreases from PILS-R0 to PILS-R1. In its turn, the extended PILS-R2 variant produces rather quantitative agreement with FCI for the first three states and a somewhat worse quality for the fourth state, $5^{1} \Sigma_{g}^{+}$(See Table II).

We finally note that again the calculations with the DMLS functional (not shown) give rather poor results. In particular, there is no DMLS excitation corresponding to the lowest FCI or PILS excitations for $R(\mathrm{H}-\mathrm{H})>3$ a.u. This is because DMLS has the fundamental deficiency that, as a genuine 1 RDM functional, it is not able in the adiabatic approximation to produce the nonzero $\delta n_{k}$ at finite $\omega$, so that it cannot describe the $2^{1} \Sigma_{g}^{+}$of the stretched $\mathrm{H}_{2}$ with its predominantly diagonal doubly excited character.

\section{APPLICATIONS: POTENTIAL ENERGY CURVES FOR THE ${ }^{1} \Sigma^{+}$EXCITED STATES OF $\mathrm{HeH}^{+}$}

The potential energy curves for the lowest excited states of the ${ }^{1} \Sigma^{+}$symmetry of the $\mathrm{HeH}^{+}$molecule are displayed in Fig. 3. $\mathrm{HeH}^{+}$is an example of a simple molecular system with $\mathrm{CT}$ excitations. Unlike the excitations in the $\mathrm{H}_{2}$ molecule discussed in Secs. V and VI, the lowest excitations in $\mathrm{HeH}^{+}$are, mainly, single excitations at all bond distances. Excitations in the stretched $\mathrm{HeH}^{+}$molecule can be described as combinations of the long-range $\mathrm{CT}$ of a single electron from $\mathrm{He}$ to $\mathrm{H}$ and a single-electron promotion to the higher, Rydberg-like orbitals of He. 


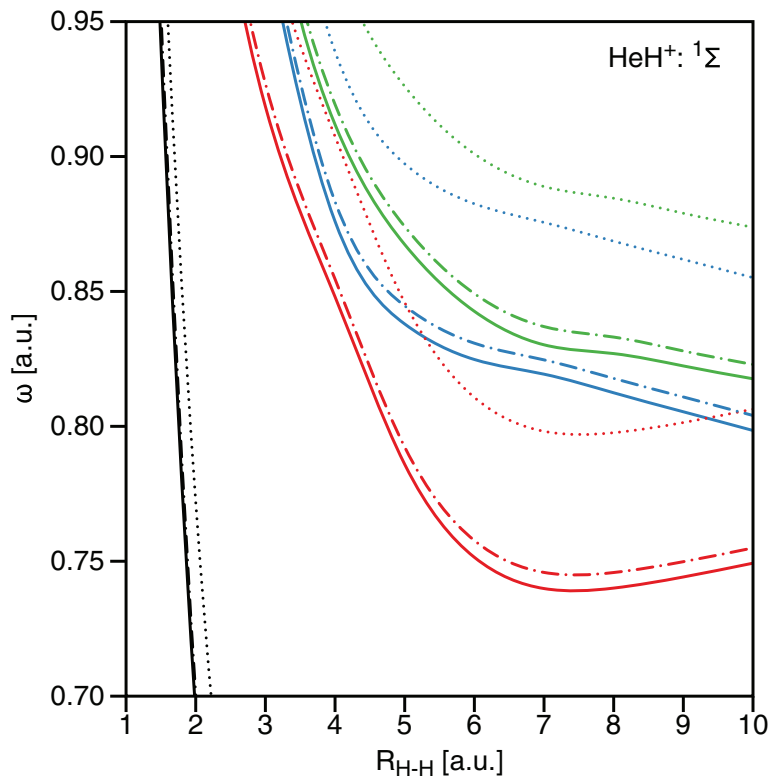

FIG. 3. Exact and PILS calculations of $\mathrm{HeH}^{+1} \Sigma^{+}$excitation energies to the 1st-4th excited states along the bond breaking coordinate. FCI: solid lines; PILS-R0: dots; PILS-R1: dotted-dashed lines. Black, red, blue, green: 1st4 th excited state, $2^{1} \Sigma^{+}-5^{1} \Sigma^{+}$.

The lowest $2^{1} \Sigma^{+}$excited state of stretched $\mathrm{HeH}^{+}$has a significantly lower energy than other states. Its PES goes down to $\sim 0.40$ a.u. and then becomes flat. It represents a relatively low-energy CT from the $1 s$ orbital of $\mathrm{He}$ to the $1 s$ orbital of $\mathrm{H}$, which no longer changes in energy beyond $\sim 4.5$ bohr. In Fig. 3 only part of the curve for this state is visible, in order to display the more interesting higher states better. The $2^{1} \Sigma^{+}$excited state is described excellently at the PILS-R1 level along the entire distance range, and even the $\mathrm{R} 0$ approximation is not so bad, just being some 0.05 a.u. too high in the asymptotic distance range. The next state, $3^{1} \Sigma^{+}$, is at $R(\mathrm{He}-\mathrm{H})=5$ a.u. a mixture of the $\mathrm{CT}$ (which has a larger contribution) to, mainly, the $2 p_{z}$ orbital of $\mathrm{H}(z$ is the molecular axis) plus a local excitation $\mathrm{He}(1 s) \rightarrow \mathrm{He}(2 s)$. The $4^{1} \Sigma^{+}$is mostly CT, $\mathrm{He}(1 s) \rightarrow \mathrm{H}(2 s, 2 p)$. And the $5^{1} \Sigma^{+}$is, again, a mixture of a local excitation (which has, this time, a larger contribution) $\mathrm{He}(1 s) \rightarrow \mathrm{He}(2 s)$ plus the CT $\mathrm{He}(1 s)$ $\rightarrow \mathrm{H}(2 s)$.

As was mentioned in the Introduction, the long-range CT presents a problem in TDDFT. ${ }^{8,14}$ In the case of a donor-acceptor CT excitation, in order to provide the proper correction to the deficient zero-order TDDFT estimate $\omega_{\alpha}^{s}$ $=\epsilon_{a}-\epsilon_{i}$, the xc kernel $f_{\mathrm{xc}}$ should diverge in the long-range limit. ${ }^{12}$ However, the present CT to the zero-electron acceptor $\mathrm{H}^{+}$, as was explained in Ref. 4, is a special case, in which the accurate $\omega_{\alpha}^{s}$ of the KS theory would be also the accurate long-range CT excitation energy $\omega_{\alpha}$. In this case, the substantial underestimation of the $\mathrm{HeH}^{+}$excitation energy observed in Ref. 4 with the standard ALDA and AGGAs of TDDFT, is due to the underestimation of the donor ionization energy ( $1 s$ orbital energy) with these approximations, which becomes particularly large for He.

In contrast, PILS is an accurate functional for stationary two-electron systems. Moreover, adiabatic PINO-PILS the-
TABLE III. Excitation energies (in hartree) for lowest $4{ }^{1} \Sigma^{+}$states of the $\mathrm{HeH}^{+}$molecule at the equilibrium bond distance.

\begin{tabular}{lcccc}
\hline \hline State & $2^{1} \Sigma^{+}$ & $3{ }^{1} \Sigma^{+}$ & $4{ }^{1} \Sigma^{+}$ & $5^{1} \Sigma^{+}$ \\
\hline PILS-R0 & 1.027 & 1.275 & 1.433 & 1.506 \\
PILS-R1 & 0.977 & 1.242 & 1.397 & 1.472 \\
PILS-R2 & 0.973 & 1.230 & 1.388 & 1.461 \\
FCI & 0.964 & 1.225 & 1.383 & 1.456 \\
\hline \hline
\end{tabular}

ory accurately describes the dynamics of two-electron systems (see Sec. II). Then, deviations of the PILS curves in Figures 1-3 are solely due to a limited number of $\delta \gamma_{k l}$ elements retained in the restricted variants PILS-R0 and PILS$\mathrm{R} 1$. It appears that the effect of restriction of the response vector, in particular the $\mathrm{R} 0$ case, are somewhat larger for $\mathrm{HeH}^{+}$, while an apparent cause such as the nondynamical correlation in elongated $\mathrm{H}_{2}$ is not obvious. The PILS-R0 variant consistently overestimates the excitation energy $\omega_{\alpha}$ of $\mathrm{HeH}^{+}$by some 0.05 a.u. It is interesting to note that all PILS-R0 curves exhibit a nearly uniform upward shift of this amount at all bond distances considered (see Fig. 3). In PILS-R1 this shift is reduced by an order of magnitude for the stretched $\mathrm{HeH}^{+}$. As can be seen in Table III the PILS-R2 variant further improves to the same level of $\sim 0.005$ a.u. as in stretched $\mathrm{H}_{2}$. Due to this, all PILS-R2 curves closely reproduce the reference FCI ones (see Fig. 3).

The DMLS-R1 curves (not shown) are reasonable in this case. One can attribute this to the predominantly singleexcitation nature of the $\mathrm{HeH}^{+1} \Sigma^{+}$states. Also for ${ }^{1} \Sigma_{u}^{+}$and ${ }^{1} \Sigma_{g}^{+}$states of $\mathrm{H}_{2}$ we have observed relatively reasonable results with DMLS-R1 for excited states with very little double excitation character. Apparently, DMLS-R1 performs better for the mostly single-excitation states, such as the present excited ${ }^{1} \Sigma^{+}$states of $\mathrm{HeH}^{+}$. Again, however, the DMLS calculations deteriorate upon extension of the response vector.

In Table III the excitation energies of the ${ }^{1} \Sigma^{+}$states calculated at the equilibrium $\mathrm{He}-\mathrm{H}^{+}$bond distance are presented. One can see in Table III the above mentioned nearly consistent overestimation of the excitation energies with the restricted PILS-R0 calculations, which is close to 0.05 a.u. at the equilibrium distance. In PILS-R1 this shift is reduced to $\sim 0.015$ a.u., which is only half the corresponding PILS-R1 shift for excitations in $\mathrm{H}_{2}$ (see Tables I and II). Again, extension to PILS-R2 substantially reduces the upward shift and produces a rather quantitative agreement with FCI.

\section{CONCLUSIONS}

In this paper the orbital excitation structure of the 1RDM response is analyzed and the adiabatic response PINO theory is applied to the calculation of the energies of bonding $\rightarrow$ antibonding (bond breaking) excitations, double excitations (both diagonal and off-diagonal), high (including Rydberg-type) excitations, and (pseudo) charge transfer excitations, along the bond-dissociation coordinate of the prototype molecules $\mathrm{H}_{2}$ and $\mathrm{HeH}^{+}$.

The interpretation of the 1RDM response in terms of the transition density matrices associated with the excited 
states is derived. It is emphasized that the $1 \mathrm{RDM}$ response encompasses both single and double excitation character of transitions to the excited states. Restricted variants of the response calculations are proposed, in which the size of the response vector is effectively reduced by retaining only single excitation as well as diagonal and a limited number of off-diagonal double-excitation 1RDM response elements. It proves to be possible to reduce the size of the density matrix response problem, which in principle has dimension of the order $\frac{1}{2} n^{2} \times \frac{1}{2} n^{2}$ with $n$ the number of basis functions, to dimensions only slightly larger than the $n_{\text {occ }} n_{\text {virt }} \times n_{\text {occ }} n_{\text {virt }}$ size of TDDFT response calculations.

The restricted adiabatic PINO theory is shown to successfully resolve problematic cases of adiabatic TDDFT. Adiabatic time-dependent PINO theory can naturally describe double excitations, which are absent in the adiabatic TDDFT. The PINO theory also successfully resolves the problem of standard TDDFT of the vanishing energy of the bond-breaking $\left(\sigma_{g} \rightarrow \sigma_{u}\right)$ excitation to the $1^{1} \Sigma_{u}^{+}$state of the $\mathrm{H}_{2}$ molecule. In part of the distance range this state is strongly ionic and this transition has pseudo CT character, which does not pose any problem. The true $\mathrm{CT}$ transitions in the stretched $\mathrm{HeH}^{+}$ molecule are also described very well. For both $\mathrm{H}_{2}$ and $\mathrm{HeH}^{+}$ the higher excited states show marked improvement when the TDDFT-like size of the problem of the R0 variant is somewhat extended in the R1 and R2 variants. These afford offdiagonal double excitations, or equivalently, in $\mathrm{H}_{2}$, excitations out of the $1 \sigma_{u}$ orbital, which becomes fractionally occupied at long distances. At the moderately extended PILS-R2 level rather quantitative agreement with the reference FCI data is obtained.

We have shown that it is important to extend TDDMFT to include further parameters, which enter the theory as phases of the orbitals. ${ }^{25,26}$ This leads to the PINO response theory applied in this work. The present results manifest the crucial importance of using in the adiabatic approach of timedependent theory orbital functionals with the proper orbital phase dependence. It appears that, unlike the PINO-PILS response calculations, the TDDMFT calculations with the density matrix functional DMLS (orbital phase invariant) fail to treat properly double excitations. Because of this, the restricted DMLS-R1 misses the lowest excited ${ }^{1} \Sigma_{g}^{+}$state of the stretched $\mathrm{H}_{2}$ which has diagonal double-excitation character. It also produces a too low-lying artificial intruder ${ }^{1} \Sigma_{u}^{+}$ state of $\mathrm{H}_{2}$ at shorter bond distances. As a matter of fact, the DMLS calculations are not stable against further extension of the size of the response problem. Upon extending the range of the $i$ index to all basis functions, the calculated spectrum then becomes totally unrealistic, with very many spurious excitations that behave erratically as a function of the distance. $^{27}$

The natural next step of the development of the PINO theory would be application of this theory to the adiabatic response calculations of systems having more than two electrons. For this, the development of an $N$-electron functional is required, which, just as the two-electron PILS functional of the present paper, would have a proper dependence on PINOs and would provide a good quality of the potential energy curves for excited states obtained with, preferably, similarly restricted calculations as explored in this paper. This work is in progress.

\section{ACKNOWLEDGMENTS}

This work has been supported by the Netherlands Foundation for Research (NWO) (KJHG and OVG), project 700-52-302 and by the World Class University program (WCU) through the Korea Science and Engineering Foundation (KOSEF) funded by the Ministry of Education, Science and Technology (Project No. R32-2008-000-10180-0) (KJHG, OVG, and EJB).

${ }^{1}$ N. T. Maitra, F. Zhang, R. J. Cave, and K. Burke, J. Chem. Phys. 120, 5932 (2004).

${ }^{2}$ J. Neugebauer and E. J. Baerends, J. Chem. Phys. 121, 6155 (2004).

${ }^{3}$ K. J. H. Giesbertz, E. J. Baerends, and O. V. Gritsenko, Phys. Rev. Lett. 101, 033004 (2008).

${ }^{4}$ K. J. H. Giesbertz, K. Pernal, O. V. Gritsenko, and E. J. Baerends, J. Chem. Phys. 130, 114104 (2009).

${ }^{5}$ I. A. Mikhailov, S. Tafur, and A. Masunov, Phys. Rev. A 77, 012510 (2008).

${ }^{6}$ O. Gritsenko, S. J. A. van Gisbergen, A. Görling, and E. J. Baerends, J. Chem. Phys. 113, 8478 (2000).

${ }^{7}$ K. J. H. Giesbertz and E. J. Baerends, Chem. Phys. Lett. 461, 338 (2008).

${ }^{8}$ A. Dreuw, J. L. Weisman, and M. Head-Gordon, J. Chem. Phys. 119, 2943 (2003).

${ }^{9}$ K. Pernal, O. Gritsenko, and E. J. Baerends, Phys. Rev. A 75, 012506 (2007).

${ }^{10}$ K. Pernal, K. Giesbertz, O. Gritsenko, and E. J. Baerends, J. Chem. Phys. 127, 214101 (2007)

${ }^{11}$ M. E. Casida, in Recent Advances in Density-Functional Methods, Part I, edited by D. P. Chong (World Scientific, Singapore, 1995), p. 155

${ }^{12}$ O. Gritsenko and E. J. Baerends, J. Chem. Phys. 121, 655 (2004).

${ }^{13}$ O. Gritsenko and E. J. Baerends, Can. J. Chem. 87, 1383 (2009).

${ }^{14}$ A. Dreuw and M. Head-Gordon, Chem. Rev. 105, 4009 (2005).

${ }^{15}$ M. Hellgren and E. K. U. Gross, e-print arXiv:1108.3100.v1.

${ }^{16}$ O. V. Gritsenko, K. Pernal, and E. J. Baerends, J. Chem. Phys. 122, 204102 (2005).

${ }^{17}$ M. Piris, Int. J. Quantum Chem. 106, 1093 (2006).

${ }^{18}$ N. N. Lathiotakis and M. Q. L. Marques, J. Chem. Phys. 128, 184103 (2008).

${ }^{19}$ M. A. L. Marques and N. N. Lathiotakis, Phys. Rev. A 77, 032509 (2008).

${ }^{20}$ N. N. Lathiotakis, N. Helbig, A. Zacarias, and E. K. U. Gross, J. Chem. Phys. 130, 064109 (2009).

${ }^{21}$ D. R. Rohr, K. Pernal, O. V. Gritsenko, and E. J. Baerends, J. Chem. Phys. 129, 164105 (2008).

${ }^{22}$ M. Piris, X. Lopez, F. Ruipérez, J. M. Matxain, and J. M. Ugalde, J. Chem. Phys. 134, 164102 (2011).

${ }^{23}$ H. Appel and E. K. U. Gross, Europhys. Lett. 92, 23001 (2010).

${ }^{24}$ R. Requist and O. Pankratov, Phys. Rev. A 83, 052510 (2011).

${ }^{25}$ K. J. H. Giesbertz, O. V. Gritsenko, and E. J. Baerends, Phys. Rev. Lett. 105, $013002(2010)$

${ }^{26}$ K. J. H. Giesbertz, O. V. Gritsenko, and E. J. Baerends, J. Chem. Phys. 133, 174119 (2010).

${ }^{27}$ K. J. H. Giesbertz, "Time-dependent one-body reduced density matrix functional theory; adiabatic approximations and beyond," $\mathrm{Ph}$.D. dissertation (Vrije Universiteit, 2010)

${ }^{28}$ E. Runge and E. K. U. Gross, Phys. Rev. Lett. 52, 997 (1984).

${ }^{29}$ G. Vignale, Phys. Rev. A 77, 062511 (2008).

${ }^{30}$ Note that we assumed here that the $\boldsymbol{A}^{+}$is positive definite. If the ground state has been taken as the reference state, this is indeed the case, since the matrix $\boldsymbol{A}^{+}$can be identified with the PINO Hessian (see Ref. 27).

${ }^{31}$ A. L. Fetter and J. D. Walecka, Quantum Theory of Many-Particle Systems (Dover, New York, 2003)

${ }^{32}$ A. M. K. Müller, Phys. Lett. A 105, 446 (1984).

${ }^{33}$ M. Buijse, "Electron correlation," Ph.D. dissertation (Vrije Universiteit, 1991)

${ }^{34}$ M. Buijse and E. J. Baerends, Mol. Phys. 100, 401 (2002).

${ }^{35}$ T. H. Dunning, Jr., J. Chem. Phys. 90, 1007 (1989).

${ }^{36}$ O. Christiansen, A. Halkier, H. Koch, P. Jørgensen, and T. Helgaker, J. Chem. Phys. 108, 2801 (1998).

${ }^{37}$ J. Wang, K. S. Kim, and E. J. Baerends, J. Chem. Phys. 135, 074111 (2011). 